\title{
Mirror Principle I
}

Bong H. Lian, ${ }^{1}$ Kefeng Liu, ${ }^{2}$ and Shing-Tung $\mathrm{Yau}^{3}$

Abstract. We propose and study the following Mirror Principle: - certain sequences of multiplicative equivariant characteristic classes on stable map moduli spaces can be computed in terms of certain hypergeometric type classes. As applications, we compute the equivariant Euler classes of obstruction bundles induced by any concavex bundles - including any direct sum of line bundles - on $\mathbf{P}^{n}$. This includes proving the formula of Candelas-de la Ossa-Green-Parkes for the instanton prepotential function for quintic in $\mathbf{P}^{4}$. We derive, among many other examples, the so-called multiple cover formula for GW invariants of $\mathbf{P}^{1}$. We also prove a formula for enumerating Euler classes which arise in the so-called local mirror symmetry for some noncompact Calabi-Yau manifolds. At the end we interprete an infinite dimensional transformation group, called the mirror group, acting on Euler data, as a certain duality group of the linear sigma model.

Appeared in Asian Journ. Math. Vol 1, No. 4 (1997).

${ }^{1}$ Department of Mathematics, Brandeis University, Waltham, MA 02154.

${ }^{2}$ Department of Mathematics, Stanford University, Stanford, CA 94305 .

${ }^{3}$ Department of Mathematics, Harvard University, Cambridge, MA 02138 . 


\section{Contents}

1. Introduction . . . . . . . . . . . . . . . . 406

1.1. The Mirror Principle . . . . . . . . . . . . . . . 406

1.2. Enumerative problems and the Mirror Conjecture . . 409

1.3. Acknowledgements . . . . . . . . . . . . . . . . 412

2. Euler Data . . . . . . . . . . . . . . . . . . . . . 413

2.1. Preliminaries and notations . . . . . . . . . . 413

2.2. Eulerity . . . . . . . . . . . . . . . . . 415

2.3. Concavex bundles . . . . . . . . . . . . . . . . 421

2.4. Linked Euler data . . . . . . . . . . . . . . . . 428

2.5. The Lagrange map and mirror transformations . . . . 431

3. Applications . . . . . . . . . . . . . . . . . . . . 437

3.1. The first convex example: The Mirror Conjecture . . 440

3.2. First concave example: multiple-cover formula . . . . 443

3.3. Second concave example: $K_{\mathbf{P}^{2}}$. . . . . . . . . . . . 444

3.4. A concavex bundle on $\mathbf{P}^{3}$. . . . . . . . . . . . . . . 445

3.5. A concavex bundle on $\mathrm{P}^{4}$. . . . . . . . . . . . . . 446

3.6. General concavex bundles . . . . . . . . . . . . . 447

3.7. Equivariant total Chern class . . . . . . . . . . . 448

3.8. Concluding remarks . . . . . . . . . . . . . . . 449

\section{Introduction}

\subsection{The Mirror Principle}

In section 2, we develop a general theory of Euler data, and give many examples. In particular we introduce the notions of a convex and a concave bundles on $\mathbf{P}^{n}$, and show that they naturally give rise to Euler data. In section 3 we apply our method to compute the equivariant Euler classes, and their nonequivariant limits, of obstruction bundles induced by a convex or a concave bundle.

We briefly outline our approach for computing multiplicative equivariant characteristic classes on stable map moduli. This outline also fixes some notations used later. Our approach is partly inspired by the idea of mirror transformations (Candelas et al), the idea of the linear sigma model (Witten, Morrison-Plesser, JinzenjiNagura,..), and the idea of using the torus action and equivariant techniques (Ellinsrud-Stromme, Kontsevich, Givental,..). All these ideas are synthesized with what we call the Mirror Principle, which we now explain. 
Let $M$ be a projective manifold and $\beta \in H^{2}(M, \mathbf{Z})$. Let $\overrightarrow{\mathcal{M}}_{g, k}(\beta, M)$ be the stable map moduli space of degree $\beta$, arithmetic genus $g$, with $k$ marked points. For a good introduction to stable maps, see [16]. Throughout this paper, we shall only deal with the case with $g=0$.

We begin by analyzing two distinguished types of fixed points under an induced torus $T$ action on $\overline{\mathcal{M}}_{0,0}\left(d, \mathbf{P}^{n}\right)$. Both types of fixed points reflect the structure of the stable map moduli space. A smooth fixed point we consider is a degree $d$ cover of a $T$-invariant $\mathbf{P}^{1}$ joining two fixed points $p_{i}, p_{j}$ in $\mathbf{P}^{n}$. A singular fixed point we consider is in the compactification divisor. It is given by gluing together two 1pointed maps $\left(f_{1}, C_{1}, x_{1}\right) \in \overline{\mathcal{M}}_{0,1}\left(r, \mathbf{P}^{n}\right)$ and $\left(f_{1}, C_{2}, x_{2}\right) \in \overline{\mathcal{M}}_{0,1}(d-$ $\left.r, \mathbf{P}^{n}\right)$ at the marked points with $f_{1}\left(x_{1}\right)=f_{2}\left(x_{2}\right)=p_{i} \in \mathbf{P}^{n}\left(p_{i}\right.$ being a $T$-fixed in $\left.\mathbf{P}^{n}\right)$, resulting in a degree $d$ stable map $(f, C)$. We consider two types of $T$-equivariant bundles $V$ on $\mathrm{P}^{n}$, which we called convex and concave respectively (Definition 2.7). To be brief, we consider the convex case in this outline. A convex bundle $V \rightarrow \mathbf{P}^{n}$ induces on $\overline{\mathcal{M}}_{0,0}\left(d, \mathbf{P}^{n}\right)$ an obstruction bundle $U_{d}$ whose fiber at $(f, C)$ is the section space $H^{0}\left(C, f^{*} V\right)$. First we have the exact sequence over C

$$
\left.0 \rightarrow f^{*} V \rightarrow f_{1}^{*} V \oplus f_{2}^{*} V \rightarrow V\right|_{p_{i}} \rightarrow 0 .
$$

Passing to cohomology, we have

$$
\left.0 \rightarrow H^{0}\left(C, f^{*} V\right) \rightarrow H^{0}\left(C_{1}, f_{1}^{*} V\right) \oplus H^{0}\left(C_{2}, f_{2}^{*} V\right) \rightarrow V\right|_{p_{i}} \rightarrow 0 .
$$

Hence we obtain a similar exact sequence for the $U_{d}$ restricted to a suitable fixed point set. Let $b_{T}$ be any multiplicative $T$-equivariant characteristic class [23] for vector bundles. The exact sequence on the fixed point set above gives rise to the identity, which we call the gluing identity:

$$
b_{T}(V) \cdot b_{T}\left(U_{d}\right)=b_{T}\left(U_{r}\right) \cdot b_{T}\left(U_{d-r}\right) .
$$

Let $M_{d}:=\overline{\mathcal{M}}_{0,0}\left((1, d), \mathrm{P}^{1} \times \mathrm{P}^{n}\right)$. This space has a $G=S^{1} \times T$ action. There is a natural equivariant contracting map $\pi: M_{d} \rightarrow$ $\overline{\mathcal{M}}_{0,0}\left(d, \mathbf{P}^{n}\right)$ given by

$$
\pi:(f, C) \in M_{d} \mapsto\left(\pi_{2} \circ f, C^{\prime}\right) \in \overline{\mathcal{M}}_{0,0}\left(d, \mathbf{P}^{n}\right)
$$

where $\pi_{2}$ denotes the projection onto the second factor of $\mathbf{P}^{1} \times \mathbf{P}^{n}$. Here $C^{\prime}=C$ if $\left(\pi_{2} \circ f, C\right)$ is still stable; and if $\left(\pi_{2} \circ f, C\right)$ is unstable, this is the case when $C$ is of the form $C=C_{1} \cup C_{0} \cup C_{2}$ with $\pi_{2} \circ f\left(C_{0}\right)$ 
a point in $\mathbf{P}^{n}$, then $C^{\prime}$ is obtained from $C$ by contracting the unstable component $C_{0}$.

Now via $\pi$, we pull back to $M_{d}$ all the information obtained above on $\overline{\mathcal{M}}_{0,0}\left(d, \mathbf{P}^{n}\right)$. The reason is that there is an collapsing map $\varphi$ : $M_{d} \rightarrow N_{d}=\mathbf{P}^{(n+1) d+n}$ which then allows us to perform computations on the linear object $N_{d}$. We call $N_{d}$ the linear sigma model and $M_{d}$ the nonlinear sigma model. There is a natural $G$ action on $N_{d}$ such that $\varphi$ is $G$-equivariant. For example, to determine an equivariant cohomology class $\omega$ on $N_{d}$, we only need to know its restrictions $\iota_{p_{i r}}^{*}(\omega)$ to the $(n+1)(d+1) G$-fixed points $\left\{p_{i r}\right\}$ in $N_{d}$. The corresponding weights of the $G$ action on $N_{d}$ are $\lambda_{i}+r \alpha$. Let $Q_{d}$ be the push-forward of $b_{T}\left(V_{d}\right)=\pi^{*} b_{T}\left(U_{d}\right)$ into $N_{d}$, ie. $Q_{d}=\varphi_{!} b_{T}\left(V_{d}\right)$. Then the classes $Q_{d}$ inherit the gluing identity (Theorem 2.8)

$$
\iota_{p_{i, 0}}^{*}\left(Q_{0}\right) \cdot \iota_{p_{i r}}^{*}\left(Q_{d}\right)=\iota_{p_{i r}}^{*}\left(Q_{r}\right) \cdot \iota_{p_{i r}}^{*}\left(Q_{d-r}\right)
$$

which is an identity in the ring $H_{G}^{*}(p t)=H^{*}(B G)$. The sequence $Q_{d}$ is an example of what we call an Euler data (Definition 2.3). We summarize the various ingredients used, now and later, in our constructions:

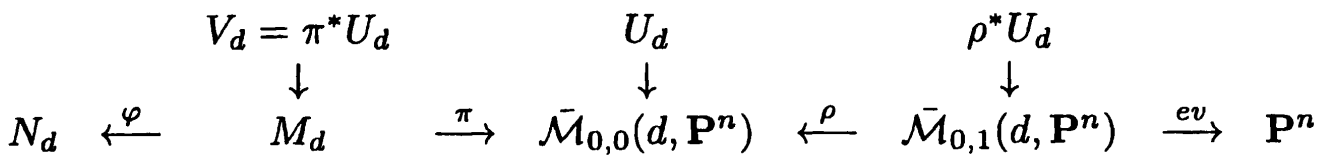

where $\rho$ forgets, and $e v$ evaluates at, the marked point of a 1-pointed stable map. Also note that $U_{d}=\rho_{!}\left(e v^{*} V\right)$, ie. the direct image of $e v^{*} V$.

The gluing identity is not enough to determine all $Q_{d}$. In order to get further information we localize the $Q_{d}$ to a fixed point in $N_{d}$ whose inverse image in $M_{d}$ is a smooth fixed point, and compute $Q_{d}$ at the special values of $\alpha=\left(\lambda_{i}-\lambda_{j}\right) / d$. The property of $b_{T}\left(U_{d}\right)$ at a smooth fixed point comes into play here. For simplicity in this outline, let's consider for example the case when $b_{T}$ is the equivariant Euler class $e_{T}$ and $V=\mathcal{O}(l)$. In this case we find that, at $\alpha=\left(\lambda_{i}-\lambda_{j}\right) / d$,

$$
\iota_{p_{i, 0}}^{*}\left(Q_{d}\right)=\prod_{m=0}^{l d}\left(l \lambda_{i}-m\left(\lambda_{i}-\lambda_{j}\right) / d\right) .
$$

This immediately tells us that the $Q_{d}$ should be compared with the sequence of classes

$$
P: \quad P_{d}=\prod_{m=0}^{l d}(l \kappa-m \alpha)
$$


(Theorem 2.10), which has $\iota_{p_{i, 0}}^{*}\left(P_{d}\right)=\iota_{p_{i, 0}}^{*}\left(Q_{d}\right)$ at $\alpha=\left(\lambda_{i}-\lambda_{j}\right) / d$. We find that $P$ is another example of an Euler data. This Euler data will then naturally give rise to a generating series of hypergeometric type, hence explains the very origin of these functions in enumerative problems on stable moduli! The same holds true for a large class of vector bundles $V$ on $\mathbf{P}^{n}$.

Finally, under a suitable bound on $c_{1}(V)$, we can completely determine the $Q_{d}$, in terms of $P_{d}$ by means of a mirror transformation argument (Theorem 3.9). We can in turn use $Q_{d}$ to compute $e_{T}\left(U_{d}\right)$ and their nonequivariant limits (Theorem 3.2). Our approach, thus, makes the roles of three objects and their relationships quite transparent: certain fixed point sets, equivariant multiplicative characteristic classes, and series of hypergeometric type. Our method works well for many characteristic classes such as the Euler class and the total Chern class.

We summarize this Mirror Principle. Let $b_{T}$ be a multiplicative equivariant characteristic class, $V$ an equivariant vector bundle on $\mathbf{P}^{n}$ which induces a sequences of vector bundles $U_{d} \rightarrow \overline{\mathcal{M}}_{0,0}\left(d, \mathbf{P}^{n}\right)$.

1. (Euler Data) The behaviour of the $U_{d}$ at a singular fixed point gives rise to the gluing identity. In turn this defines an Euler data $Q_{d}$ on the linear sigma model $N_{d}$.

2. (Linking) The behaviour of the $U_{d}$ at a smooth fixed point allows us to read off the restrictions of $Q_{d}$ at certain fixed points of $N_{d}$ for special values of weights. The restriction values determine a distinguished Euler data $P_{d}$ to be compared with the $Q_{d}$.

3. (Mirror Transformations) Compute the $Q_{d}$ and the $b_{T}\left(U_{d}\right)$ in terms of the $P_{d}$ explicitly by means of a mirror transformation argument.

Our approach outlined here can be applied to a rather broad range of cases by replacing $\mathbf{P}^{n}$ by other manifolds. They include manifolds with torus action and their submanifolds. We will study the cases of toric varieties and homogeneous manifolds in a forth-coming paper [37]. On the other hand, we can even go beyond equivariant multiplicative characteristic classes. In a future paper, we will study a sequence of equivariant classes $T_{d}$ of geometric origin on stable moduli satisfying our gluing identity.

\subsection{Enumerative problems and the Mirror Conjecture}

For the remarkable history of the Mirror Conjecture, see [14]. In 1990, Candelas, de la Ossa, Green, and Parkes [8] conjectured a formula for counting the number $n_{d}$ of rational curves in every degree $d$ on a general quintic in $\mathbf{P}^{4}$. The conjectured formula gives a 
generating function for the $n_{d}$ explicitly in terms of certain hypergeometric functions. Their computation is based on the existence of "mirror manifolds", conjectured by a number of physicists including Dixon and Lerche, Vafa and Warner on the basis of physical intuition. Mirror symmetry took a dramatic turn upon the appearance of the papers of Greene-Plesser [21] and of Candelas et al [8]. In [21], they established the existence of mirror manifolds for a particular class of Calabi-Yau manifolds including the quintic.

On the mathematical side, it has been conjectured earlier by Clemens that the number of rational curves in every degree in a general quintic is finite. The conjectured formula agrees with a classical result in degree 1 , an earlier computation by $\mathrm{S}$. Katz in degree 2, and has been verified in degree 3 by Ellingsrud-Stromme. In 1994, using results of Uhlenbeck-Sacks, Gromov, Parker-Wolfson, and Witten, Ruan-Tian introduced the notions of $J$-holomorphic maps and symplectic Gromov-Witten (GW) invariants. Motivated by the same works, Kontsevich proposed an algebraic geometric analogue of GW invariants and stable maps. The latter is the algebraic geometric counterpart of $J$-holomorphic maps. Generalizations have been given by Li-Tian and Behrend-Fentachi. A recent paper of Li-Tian shows that the symplectic version and the algebraic geometric version of the GW theory are essentially the same in the projective category. Beautiful applications of ideas from quantum cohomology and GW theory have recently been done by Caporaso-Harris [10], Crauder-Miranda [11], DiFrancesco-Itzykson [12], Bryan-Leung [2], and others solving many important enumerative problems.

Closer to mirror symmetry, it is known that the degree $k \mathrm{GW}$ invariants for $\mathbf{P}^{1}$ (the so-called multiple-cover contribution) is given by $k^{-3}$. This was conjectured in [8], justified in [1][44] using a different compactification, and in [38] using the stable map compactification. According to [34], the number

$$
K_{d}=\sum_{k \mid d} n_{d / k} k^{-3}
$$

is the degree of the Euler class $c_{\text {top }}\left(U_{d}\right)$ for $U_{d} \rightarrow \overline{\mathcal{M}}_{0,0}\left(d, \mathbf{P}^{4}\right)$ induced by $\mathcal{O}(5) \rightarrow \mathbf{P}^{4}$.

By means of the torus action on $\mathbf{P}^{4}$, Ellingsrud-Stromme [15] and Kontsevich [34] have verified that $n_{3}$ and $n_{4}$ respectively agree with the conjectured formula. In some recent papers [18][19], Givental introduces some ideas which emphasize the use of equivariant version of quantum cohomology (see also Kim [27]). Unfortunately, the proof in [18]for the formula of Candelas et al, which has been read by many 
prominent experts, is incomplete. In light of our present paper, it is now clear that essentially all ingredients (equivariant cohomology theory, the Atiyah-Bott formula, equivariant Euler classes, obstruction bundles, fixed point structures, the linear sigma model, the graph construction, hypergeometric series, mirror transformations, etc) required to prove the formula of Candelas et al have all been well-known to experts since at least 1995. Thus the remaining nontrivial task, is to set up and put together in details all the pieces in a correct way to give a complete proof. In our present paper, we have introduced the substantial new ideas (beyond the known ingredients) needed to give the first complete proof. It is conceivable that our new ideas here can furnish the necessary parts missing in the paper [18]. More recently, fixed point method has also been applied by Graber-Pandharipande [20] to study GW invariants of $\mathbf{P}^{n}$.

We emphasize that the formula of Candelas et al, albeit conjectural, not only computes the $K_{d}$ in terms of hypergeometric functions, but also provides a crucial guide for putting together various technical inputs in our proof. While applying the Atiyah-Bott localization formula on the stable moduli and on the linear sigma model yield important pieces of information about the cohomology classes in question, it is the formula of Candelas et al which provides the clues for how to put the pieces together - in terms of hypergeometric functions (which are periods of a holomorphic 3-form on a mirror manifold). Earlier attempts on the conjectured formula using quantum cohomology seems to have confused the issue considerably.

We believe that the machinery introduced in this paper will be useful for many other enumerative problems, aside from proving the formula of Candelas et al. In fact we have applied our machinery to problems in local mirror symmetry proposed by C.Vafa, S. Katz, and others. ${ }^{4}$

We now formulate one of our main theorems in this paper. Let

$$
K_{d}=\int_{\overline{\mathcal{M}}_{0,0}\left(d, \mathbf{P}^{4}\right)} c_{t o p}\left(U_{d}\right), \quad F(T)=\frac{5 T^{3}}{6}+\sum_{d>0} K_{d} e^{d T}
$$

Consider the fourth order hypergeometric differential operator [8]:

$$
L:=\left(\frac{d}{d t}\right)^{4}-5 e^{t}\left(5 \frac{d}{d t}+1\right) \cdots\left(5 \frac{d}{d t}+4\right) .
$$

S. Katz has informed us that A. Elezi has also studied a similar problem. 
By the Frobenius method, it is easy to show that

$$
f_{i}:=\left.\frac{1}{i !}\left(\frac{d}{d H}\right)^{i}\right|_{H=0} \sum_{d \geq 0} e^{d(t+H)} \frac{\prod_{m=1}^{5 d}(5 H+m)}{\prod_{m=1}^{d}(H+m)^{5}}, \quad i=0,1,2,3,
$$

form a basis of solutions to the differential equation $L \cdot f=0$. Let

$$
T=\frac{f_{1}}{f_{0}}, \quad \mathcal{F}(T)=\frac{5}{2}\left(\frac{f_{1}}{f_{0}} \frac{f_{2}}{f_{0}}-\frac{f_{3}}{f_{0}}\right) .
$$

Theorem 1.1. (The Mirror Conjecture) $F(T)=\mathcal{F}(T)$.

The transformation on the functions $f_{i}$ given by the normalization

$$
f_{i} \mapsto \frac{f_{i}}{f_{0}}
$$

and the change of variables

$$
t \mapsto T(t)=\frac{f_{1}}{f_{0}}
$$

are known as the mirror transformation. By the construction of Candelas et al, the functions $f_{0}, . ., f_{3}$ are periods of a family of Calabi-Yau threefolds. By the theorem of Bogomolov-Tian-Todorov, these periods in fact determine the complex structure of the threefold.

A similar Mirror Conjecture formula holds true for a three dimensional Calabi-Yau complete intersection in a toric Fano manifold [37]. This will turn out to agree with the many beautiful mirror symmetry computations [40][36][5][9][4][30][31].

In this paper, to make the ideas clear we restrict ourselves to the simplest case, genus 0 stable moduli space for $\mathbf{P}^{n}$. In a forth-coming paper [37], we extend our discussions to toric varieties, homogeneous manifolds, and general projective manifolds. We also hope to eventually understand from our point of view the far reaching results for higher genus of [6], and the beautiful computations [17][13] for elliptic GW invariants.

\subsection{Acknowledgements}

We thank A. Todorov, A. Strominger, C. Vafa for helpful discussions. Our special thanks are due to $\mathrm{J}$. Li who has been very helpful throughout our project. 


\section{Euler Data}

One of the key ingredients in our approach is the linear sigma model, first introduced by Witten [45], and later used to study mirror symmetry by Morrison-Plesser [29], Jinzenji-Nagura [25], and others, resulting in new insights into the origin of hypergeometric series. In this paper, we consider the $S^{1} \times T$-equivariant cohomology of the linear sigma model.

\subsection{Preliminaries and notations}

Let $T$ be an $r$-dimensional real torus with a complex linear representation on $\mathbf{C}^{N+1}$. Let $\beta_{0}, . ., \beta_{N}$ be the weights of this action. We consider the induced action of $T$ on $\mathbf{P}^{N}$, and the $T$-equivariant cohomology with coefficients in $\mathbf{Q}$, which we shall denote by $H_{T}^{*}(-)$. Now $H_{T}^{*}(p t)$ is a polynomial algebra in $r$-variables, and $\beta_{i}$ may be regarded as elements of $H_{T}^{2}(p t)$. Throughout this paper, we shall follow the convention that such generators have degree 1 . It is known that the equivariant cohomology of $\mathbf{P}^{N}$ is given by [32]

$$
H_{T}\left(\mathbf{P}^{N}\right)=H_{T}(p t)[\zeta] /\left(\prod_{i=0}^{N}\left(\zeta-\beta_{i}\right)\right) .
$$

Here $\zeta$, which we shall call the equivariant hyperplane class, is a fixed lifting of the hyperplane class of $\mathbf{P}^{N}$. Each one-dimensional weight space in $\mathbf{C}^{N+1}$ becomes a fixed point $p_{i}$ in $\mathbf{P}^{N}$. We shall identify the rings $H_{T}^{*}\left(p_{i}\right)$ and $H_{T}^{*}(p t)=H^{*}(B T)$. There are $N+1$ canonical restriction maps $\iota_{p_{i}}^{*}: H_{T}\left(\mathbf{P}^{N}\right) \rightarrow H_{T}(p t)$, given by $\zeta \mapsto \beta_{i}, i=$ $0, . ., N$. There is also a push-forward map $H_{T}\left(\mathbf{P}^{N}\right) \rightarrow H_{T}(p t)$ given by integration over $\mathbf{P}^{N}$. By the localization formula, it is given by

$$
\omega \mapsto \int_{\mathbf{P}^{N}} \omega=\operatorname{Res}_{\zeta} \frac{\omega}{\prod_{i=0}^{N}\left(\zeta-\beta_{i}\right)} .
$$

Two situations arise frequently in this paper. First consider the standard action of $T=\left(S^{1}\right)^{n+1}$ on $\mathbf{C}^{n+1}$, and let $\lambda=\left(\lambda_{0}, . ., \lambda_{n}\right)$ denote the weights. On $\mathbf{P}^{n}$, there are $n+1$ isolated fixed points $p_{0}, . ., p_{n}$. We shall denote the equivariant hyperplane class by $p$, the canonical restrictions by $\iota_{p_{i}}^{*}: \omega \mapsto \iota_{p_{i}}^{*}(\omega)=\omega\left(\lambda_{i}\right)$, and the pushforward by

$$
p f: H_{T}\left(\mathbf{P}^{n}\right) \rightarrow H_{T}(p t)=\mathbf{Q}[\lambda] \text {. }
$$

We shall use the evaluation map $\lambda \mapsto 0$ on the ring $H_{T}^{*}\left(\mathbf{P}^{n}\right)$, and shall call this the nonequivariant limit. In this limit, $p$ becomes the ordinary hyperplane class $H \in H^{*}\left(\mathbf{P}^{n}\right)$. 
We now consider the second situation. For each $d=0,1,2, .$. , consider the following complex linear action of the group $G:=S^{1} \times T$ on $\mathbf{C}^{(n+1)(d+1)}$. We let the group act on the (ir)-th coordinate line in $\mathbf{C}^{(n+1)(d+1)}$ by the weights $\lambda_{i}+r \alpha\left(\lambda_{i}\right.$ being the weights of $T$ as before, and $\alpha$ being a weight of $\left.S^{1}\right), i=0, . ., n, r=0, . ., d$. Thus there are $(n+$ $1)(d+1)$ isolated fixed points $p_{i r}$ on the projective space $\mathbf{P}^{(n+1) d+n}$, given by those coordinate lines. In this case, we shall denote the equivariant hyperplane class by $\kappa$, the canonical restrictions by $\iota_{p_{i r}}^{*}$ : $\omega \mapsto \iota_{p_{i r}}^{*}(\omega)=\omega\left(\lambda_{i}+r \alpha\right)$, and the push-forward by

$$
p f_{d}: H_{G}\left(\mathbf{P}^{(n+1) d+n}\right) \rightarrow H_{G}(p t)=\mathbf{Q}[\alpha, \lambda] .
$$

Here we have abused the notation $\kappa$, using it to represent a class in $H_{G}\left(\mathbf{P}^{(n+1) d+n}\right)$ for every $d$. But it should present no confusion in the context it arises.

Let $N_{d}$ be the space of nonzero $(n+1)$-tuple of degree $d$ homogeneous polynomials in two variables $w_{0}, w_{1}$, modulo scalar. There is a canonical way to identify $N_{d}$ with $\mathbf{P}^{(n+1) d+n}$. Namely, a point $z \in \mathbf{P}^{(n+1) d+n}$ corresponds to the polynomial tuple $\left[\sum_{r} z_{0 r} w_{0}^{r} w_{1}^{d-r}, \ldots\right.$, $\left.\sum_{r} z_{n r} w_{0}^{r} w_{1}^{d-r}\right] \in N_{d}$. This identification will be used throughout this paper. The natural $T$-action on $(n+1)$-tuples together with the $S^{1}$ action on $\left[w_{0}, w_{1}\right] \in \mathbf{P}^{1}$ by weights $(\alpha, 0)$, induces a $S^{1} \times T$-action on $N_{d}$ given by $\left[f_{0}\left(w_{0}, w_{1}\right), . ., f_{n}\left(w_{0}, w_{1}\right)\right] \mapsto\left[e^{\lambda_{0}} f_{0}\left(e^{\alpha} w_{0}, w_{1}\right), . .\right.$, $\left.e^{\lambda_{n}} f_{n}\left(e^{\alpha} w_{0}, w_{1}\right)\right]$. This coincides with the $S^{1} \times T$-action on $\mathbf{P}^{(n+1) d+n}$ described earlier.

Definition 2.1. (Notations) We call the sequence of projective spaces $\left\{N_{d}\right\}$ the linear sigma model for $\mathbf{P}^{n}$. Here are some frequently used notations: $G:=S^{1} \times T, \mathcal{R}:=\mathbf{Q}(\lambda)[\alpha], \mathcal{R}^{-1}:=\mathbf{Q}(\lambda, \alpha), \mathcal{R} H_{G}^{*}\left(N_{d}\right):=$ $H_{G}^{*}\left(N_{d}\right) \otimes_{\mathrm{Q}[\lambda, \alpha]} \mathcal{R}, \mathcal{R}^{-1} H_{G}^{*}\left(N_{d}\right):=H_{G}^{*}\left(N_{d}\right) \otimes_{\mathbf{Q}[\lambda, \alpha]} \mathcal{R}^{-1}$, and $\operatorname{deg}_{\alpha} \omega$ means the degree in $\alpha$ of $\omega \in \mathcal{R}$.

Obviously the maps $\iota_{p_{i r}}^{*}, p f_{d}$ defined linearly over $\mathbf{Q}[\lambda, \alpha]$, can be extended $\mathcal{R}$ - or $\mathcal{R}^{-1}$-linearly. There are two natural equivariant maps between the $N_{d}$, given by

$$
\begin{aligned}
& I: N_{d-1} \rightarrow N_{d}, \quad\left[f_{0}, . ., f_{n}\right] \mapsto\left[w_{1} f_{0}, . ., w_{1} f_{n}\right] \\
& -: N_{d} \rightarrow N_{d}, \quad\left[f_{0}\left(w_{0}, w_{1}\right), . ., f_{n}\left(w_{0}, w_{1}\right)\right] \mapsto\left[f_{0}\left(w_{1}, w_{0}\right), . ., f_{n}\left(w_{1}, w_{0}\right)\right] .
\end{aligned}
$$

The second map induces on equivariant cohomology $\mathcal{R}^{-1} H_{G}^{*}\left(N_{d}\right), \bar{\kappa}=$ $\kappa-d \alpha, \bar{\alpha}=-\alpha, \bar{\lambda}_{i}=\lambda_{i}$. In particular any $x \in \mathcal{R}$ has the form 
$x=x_{-}+x_{+}$with $\bar{x}_{ \pm}= \pm x_{ \pm}$. We also extend - to the power series ring $\mathcal{R}\left[\left[e^{t}\right]\right]$ by leaving $t$ invariant.

Composing a chain of $d I$ 's, we get a canonical inclusion $N_{0}=$ $\mathbf{P}^{n} \stackrel{I_{d}}{\longrightarrow} N_{d}$. Note that the image of the fixed point $p_{i}$ is $p_{i, 0}$. For $\omega \in$ $\mathcal{R}^{-1} H_{G}^{*}\left(N_{d}\right)$, we shall denote by $I_{d}^{*}(\omega) \in \mathcal{R}^{-1} H_{G}^{*}\left(N_{0}\right)$ the restriction of $\omega$ to $N_{0}$. Since $S^{1}$ acts trivially on $\mathbf{P}^{n}$, we can write

$$
H_{G}^{*}\left(N_{0}\right)=H_{T}^{*}\left(\mathbf{P}^{n}\right)[\alpha] .
$$

In particular note that $H_{T}^{*}\left(\mathbf{P}^{n}\right)$ is invariant under ${ }^{-}$, and that $I_{d}^{*}(\kappa)=$ $p$.

Obviously the set of classes $\omega \in H_{T}^{*}\left(\mathbf{P}^{n}\right)$ with $\iota_{p_{i}}^{*}(\omega) \neq 0$ for all $i$, is closed under multiplication. We localize the $\operatorname{ring} H_{T}^{*}\left(\mathbf{P}^{n}\right)$ by allowing to invert such elements $\omega$. We denote the resulting ring by $H_{T}^{*}\left(\mathbf{P}^{n}\right)^{-1}$.

Definition 2.2. (Notations) The degree in $\alpha$ of a class $\omega \in$ $H_{T}^{*}\left(\mathbf{P}^{n}\right)^{-1}[\alpha]$ will be denoted by $\operatorname{deg}_{\alpha} \omega$. A class $\Omega \in H_{T}^{*}\left(\mathbf{P}^{n}\right)^{-1}$ with $\iota_{p_{i}}^{*}(\Omega) \neq 0$ for all $i$ will be called invertible. Throughout this paper, $\Omega$ will denote a fixed but arbitrary invertible class. $\mathcal{S}$ denotes the set of sequences of cohomology classes $Q: Q_{d} \in \mathcal{R}^{-1} H_{G}^{*}\left(N_{d}\right), \quad d=1,2, \ldots$

2.2. Eulerity

Definition 2.3. We call a sequence $Q: Q_{d} \in \mathcal{R} H_{G}^{*}\left(N_{d}\right), \quad d=$ $1,2, \ldots$, an $\Omega$-Euler data if for all $d$, and $r=0, . ., d, i=0, . ., n$,

$$
(*) \quad \iota_{p_{i}}^{*}(\Omega) \iota_{p_{i, r}}^{*}\left(Q_{d}\right)=\overline{\iota_{p_{i, 0}}^{*}\left(Q_{r}\right)} \iota_{p_{i, 0}}^{*}\left(Q_{d-r}\right),
$$

where $Q_{0}:=\Omega$. We denote by $\mathcal{A}^{\Omega}$ the set of $\Omega$-Euler data.

When dealing only with one fixed class $\Omega$ at a time, we shall say Euler rather than $\Omega$-Euler, and shall write $\mathcal{A}$ for the set of Euler data. More explicitly, condition $\left(^{*}\right)$ can be written as $\Omega\left(\lambda_{i}\right) Q_{d}\left(\lambda_{i}+\right.$ $r \alpha)=\overline{Q_{r}\left(\lambda_{i}\right)} Q_{d-r}\left(\lambda_{i}\right)$. Applying this at $r=d$, we find that

$$
Q_{d}\left(\lambda_{i}+d \alpha\right)=\overline{Q_{d}\left(\lambda_{i}\right)} .
$$

Putting $\alpha=\left(\lambda_{j}-\lambda_{i}\right) / d$, we see that $Q_{d}\left(\lambda_{j}\right)$ at $\alpha=\left(\lambda_{j}-\lambda_{i}\right) / d$ coincides with $Q_{d}\left(\lambda_{i}\right)$ at $\alpha=\left(\lambda_{i}-\lambda_{j}\right) / d$. Applying both $\left(^{*}\right)$ and (2.1) at $\alpha=\left(\lambda_{j}-\lambda_{i}\right) / r$ (hence $\lambda_{j}=\lambda_{i}+r \alpha$ ), we get

$$
\Omega\left(\lambda_{i}\right) Q_{d}\left(\lambda_{j}\right)=Q_{r}\left(\lambda_{j}\right) Q_{d-r}\left(\lambda_{i}\right) \text { at } \alpha=\left(\lambda_{j}-\lambda_{i}\right) / r .
$$


Lemma 2.4. (Reciprocity Lemma) If $Q$ is an Euler data, then for $i, j=0, . ., n, r=0,1, . ., d, d=0,1,2, . .$, we have

(i) $Q_{d}\left(\lambda_{i}+d \alpha\right)=\overline{Q_{d}\left(\lambda_{i}\right)}$.

(ii) $Q_{d}\left(\lambda_{j}\right)$ at $\alpha=\left(\lambda_{j}-\lambda_{i}\right) / d$ coincides with $Q_{d}\left(\lambda_{i}\right)$ at $\alpha=\left(\lambda_{i}-\right.$ $\left.\lambda_{j}\right) / d$ for $d \neq 0$.

(iii) $\Omega\left(\lambda_{i}\right) Q_{d}\left(\lambda_{j}\right)=Q_{r}\left(\lambda_{j}\right) Q_{d-r}\left(\lambda_{i}\right)$ at $\alpha=\left(\lambda_{j}-\lambda_{i}\right) / r$ for $r \neq 0$.

Example 1. Let $l$ be a positive integer. Put

$$
P: \quad P_{d}=\prod_{m=0}^{l d}(l \kappa-m \alpha) \in H_{G}^{*}\left(N_{d}\right) .
$$

That this is an $l p$-Euler data follows from the identity:

$$
l \lambda_{i} \prod_{m=0}^{l d}\left(l \lambda_{i}+(r-m) \alpha\right)=\prod_{m=0}^{l r}\left(l \lambda_{i}+m \alpha\right) \times \prod_{m=0}^{l(d-r)}\left(l \lambda_{i}-m \alpha\right) .
$$

This will arise naturally in the problem of computing the equivariant Euler classes of the obstruction bundles induced by $\mathcal{O}(l) \rightarrow \mathbf{P}^{n}$. (See below.)

Example 2. Put $\Omega=p^{-2}$, and

$$
P: \quad P_{d}=\prod_{m=1}^{d-1}(\kappa-m \alpha)^{2} \in H_{G}^{*}\left(N_{d}\right)
$$

This Euler data will arise in the problem of computing the so-called multiple cover contributions, ie. the GW invariants for $\mathbf{P}^{1}$.

Example 3. Put $\Omega=(-3 p)^{-1}$, and

$$
P: \quad P_{d}=\prod_{m=1}^{3 d-1}(-3 \kappa+m \alpha) \in H_{G}^{*}\left(N_{d}\right) .
$$

This Euler data will arise in the problem of computing the equivariant Euler classes of the obstruction bundles induced by the canonical bundle of $\mathbf{P}^{2}$.

Example 4. Put $\Omega=-1$, and

$$
P: \quad P_{d}=\prod_{m=0}^{2 d}(2 \kappa-m \alpha) \times \prod_{m=1}^{2 d-1}(-2 \kappa+m \alpha) \in H_{G}^{*}\left(N_{d}\right) .
$$


This Euler data will arise in the problem of computing the equivariant Euler classes of the obstruction bundles induced by $\mathcal{O}(2) \oplus \mathcal{O}(-2)$ on $\mathbf{P}^{3}$.

Example 5. It is easy to show that if $Q$ is $\Omega$-Euler and $a \in \mathbf{Q}(\lambda)$ is any nonzero element, then the data $Q^{\prime}: Q_{d}^{\prime}=a \cdot Q_{d}$ is $a \Omega$-Euler. Similarly, the data $Q^{\prime}: Q_{d}^{\prime}=(-1)^{d+1} Q_{d}$ is also $-\Omega$-Euler.

Example 6. We observe that the set of Euler data is a monoid, ie. it is closed under the product $Q_{d} Q_{d}^{\prime}$, and has the unit given by $Q_{d}=1$ for all $d$. Hence the product of an $\Omega$-Euler with an $\Omega^{\prime}$-Euler data is an $\Omega \Omega^{\prime}$-Euler data. In the geometrically setting, this multiplicative property sometimes corresponds to taking intersection of two suitable projective manifolds. In this case, the class $\Omega \in H_{T}^{*}\left(\mathbf{P}^{n}\right)$ plays the role of the equivariant Thom class of the normal bundle of such a projective manifold.

Example 7. Let $Q_{d}=\kappa(\kappa-d \alpha) \in H_{G}^{*}\left(N_{d}\right)$. Then it is again trivial to check that $Q$ is $\kappa^{2}$-Euler.

Example 8. Let $Q$ be an $\Omega$-Euler data, and $Q^{\prime}$ be an $\Omega^{\prime}$-Euler data. Suppose $Q_{d} / Q_{d}^{\prime} \in \mathcal{R} H_{G}^{*}\left(N_{d}\right)$ for all $d>0$. Then it is immediate that they form a sequence, denoted by $Q / Q^{\prime}$, which is $\Omega / \Omega^{\prime}$ - Euler. As a special case, let $Q_{d}=l^{2} \kappa(\kappa-d \alpha)$ as in Example 7, and let $P$ as in Example 1. Then $P / Q$ is a $(l p)^{-1}$-Euler data. Example 2 is obtained by squaring this. Examples 3 and 4 can also be obtained in a similar way.

Example 9. Introduce a formal variable $x$. We can extend everything above by adjoining $x$, ie. by replacing the ground field $\mathbf{Q}$ by $\mathbf{Q}(x)$. For example, it is easy to show that

$$
P: \quad P_{d}=\prod_{m=0}^{l d}(x+l \kappa-m \alpha) \in H_{G}^{*}\left(N_{d}\right)(x)
$$

satisfies the gluing identity as in Example 1, thus is an Euler data in a more general sense. Such Euler data will appear in the computations of equivariant total Chern classes.

Example 10. Let $M_{d}^{0}:=\mathcal{M}_{0,0}\left((1, d), \mathbf{P}^{1} \times \mathbf{P}^{n}\right)$ be the moduli space of holomorphic maps $\mathbf{P}^{1} \rightarrow \mathbf{P}^{1} \times \mathbf{P}^{n}$ of bidegree $(1, d)$. Recall that $M_{d}$ is the stable map compactification of $M_{d}^{0}$. Each map $f \in M_{d}^{0}$ can be represented by $f:\left[w_{0}, w_{1}\right] \mapsto\left[w_{1}, w_{0}\right] \times$ $\left[f_{0}\left(w_{0}, w_{1}\right), . ., f_{n}\left(w_{0}, w_{1}\right)\right]$, where $f_{i}$ are degree $d$ homogeneous polynomials. So there is an obvious map

$$
\varphi: M_{d}^{0} \rightarrow N_{d}, f \mapsto\left[f_{0}, . ., f_{n}\right]
$$

which is $G=S^{1} \times T$ equivariant. For convenience we define $M_{0}:=$ $N_{0}=\mathbf{P}^{n}$. With a bit of work (see below), it can be shown that 
the map $\varphi$ has an equivariant regular extension to $\varphi: M_{d} \rightarrow N_{d}$. Let $(f, C) \in M_{d}$. Then $C$ is an arithmetic genus 0 curve of the form $C=C_{0} \cup C_{1} \cup \cdots \cup C_{N}$ such that $\pi_{1} \circ f: C_{0} \stackrel{\sim}{\rightarrow} \mathrm{P}^{1}$, where $\pi_{1}, \pi_{2}$ are projections from $\mathbf{P}^{1} \times \mathbf{P}^{n}$ to the first and second factors. Each $C_{j}, j>0$, is glued to $C_{0}$ at some point $x_{j} \in C_{0}$. The map $\pi_{2} \circ f: C_{j} \rightarrow \mathbf{P}^{n}$ is of degree $d_{j}$ with $\sum_{j} d_{j}=d$, and $\pi_{1} \circ f: C_{j} \rightarrow$ $\mathbf{P}^{1}$ is constant map with image $\pi_{1} \circ f\left(x_{j}\right) \in \mathbf{P}^{1}$. If we denote by $\left[\sigma_{0}, \cdots, \sigma_{n}\right]$ the degree $d_{0}$ polynomials representing $\pi_{2} \circ f: C_{0} \rightarrow \mathbf{P}^{n}$, then $\varphi:(f, C) \mapsto\left[\sigma_{0} g, \cdots, \sigma_{n} g\right]$, where $g=\prod_{j}\left(a_{j} w_{0}-b_{j} w_{1}\right)^{d_{j}}$ with $\pi_{1} \circ f\left(x_{j}\right)=\left[a_{j}, b_{j}\right]$. Thus $\varphi$ collapses all but one component of $C$.

The idea of using a collapsing map relating two moduli problems is not new. The map $\varphi$ was known to Tian [43], and a similar map also appeared in [35] in which a collapsing map was used to relate two moduli spaces. The map $\varphi$ was also used in [18]. Similar maps have also been studied in [24][26].

Let $V=\mathcal{O}(l)$ for $l>0$, and consider the induced bundle $U_{d} \rightarrow$ $\overline{\mathcal{M}}_{0,0}\left(d, \mathbf{P}^{n}\right)$. Pulling this back via the projection $\pi$, we get a bundle $V_{d} \rightarrow M_{d}$ (see Introduction). Let $e_{T}\left(V_{d}\right)$ be the equivariant Euler class of $V_{d}$, and let $\varphi_{!} e_{T}\left(V_{d}\right)$ be its push-forward via $\varphi$. The following theorem will be a special case of a general theorem proved in the next subsection.

Theorem 2.5. The sequence $\varphi_{!} e_{T}\left(V_{d}\right) \in H_{G}^{*}\left(N_{d}\right)$ above is an lpEuler data.

We now return to the map $\varphi$. The reader who wishes to skip technical details can safely omit the proof.

Lemma 2.6. There exists a morphism $\varphi: M_{d} \rightarrow N_{d}$. Moreover $\varphi$ is equivariant with respect to the induced action of $S^{1} \times T$.

Proof: The following proof is given by J. Li. Let $M_{d}$ be the moduli space of stable morphisms $f: C \rightarrow \mathbf{P}^{1} \times \mathbf{P}^{n}$ from arithmetic genus 0 curves to $\mathbf{P}^{1} \times \mathbf{P}^{n}$ of bi-degree $(1, d)$, and let $N_{d}$ be the space of equivalence classes of $(n+1)$-tuples $\left(f_{0}, \ldots, f_{n}\right)$, where $f_{i}$ are degree $d$ homogeneous polynomials in two variables, and $\left(f_{0}, \ldots, f_{n}\right) \sim\left(f_{0}^{\prime}, \ldots, f_{n}^{\prime}\right)$ if there is a constant $c \neq 0$ such that $f_{i}=c \cdot f_{i}^{\prime}$ for all $i$. We first define the morphism $\varphi: M_{d} \rightarrow N_{d}$. For convenience, we let $\mathcal{S}$ be the category of all schemes of finite type (over $\mathbf{C}$ ) and let

$$
\mathcal{F}: \mathcal{S} \longrightarrow(\text { Set })
$$

be the the contra-variant functor that send any $S \in \mathcal{S}$ to the set of families of stable morphisms

$$
F: \mathcal{X} \longrightarrow \mathbf{P}^{1} \times \mathbf{P}^{n} \times S
$$


over $S$, where $\mathcal{X}$ are families of connected arithmetic genus 0 curves, modulo the obvious equivalence relation. Note that $\mathcal{F}$ is represented by the moduli stack $M_{d}$. Hence to define $\varphi$ it suffices to define a transformation

$$
\Psi: \mathcal{F} \longrightarrow \operatorname{Mor}\left(-, N_{d}\right)
$$

We now define such a transformation. Let $S \in \mathcal{S}$ and let $\xi \in \mathcal{F}(S)$ be represented by $(\mathcal{X}, F)$. We let $p_{i}$ be the composite of $F$ with the $i$-th projection of $\mathbf{P}^{1} \times \mathbf{P}^{n} \times S$ and let $p_{i j}$ be the composite of $F$ with the projection from $\mathbf{P}^{1} \times \mathbf{P}^{n} \times S$ to the product of its $i$-th and $j$-th components. We consider the sheaf $p_{2}^{*} \mathcal{O}_{\mathbf{p}^{n}}(1)$ on $\mathcal{X}$ and its direct image sheaf

$$
\mathcal{L}_{\xi}=p_{13 *} p_{2}^{*} \mathcal{O}_{\mathbf{P}^{n}}(1)
$$

We claim that $\mathcal{L}_{\xi}$ is flat over $S$. Indeed, by argument in the proof of Theorem 9.9 in [22], it suffices to show that $\pi_{S^{*}}\left(\mathcal{L}_{\xi} \otimes \pi_{\mathbf{P}^{1}}^{*} \mathcal{O}_{\mathbf{P}^{1}}(m)\right)$ are locally free sheaves of $\mathcal{O}_{S}$-modules for $m \gg 0$, where $\pi_{\mathbf{P}^{1}}$ and $\pi_{S}$ are the first and the second projections of $\mathbf{P}^{1} \times S$. Clearly, this sheaf is isomorphic to $p_{3^{*}}\left(p_{2}^{*} \mathcal{O}_{\mathbf{P}^{n}}(1) \otimes p_{1}^{*} \mathcal{O}_{\mathbf{P}^{1}}(m)\right)$, which is locally free because

$$
R^{i} p_{3 *}\left(p_{2}^{*} \mathcal{O}_{\mathbf{P}^{n}}(1) \otimes p_{1}^{*} \mathcal{O}_{\mathbf{P}^{1}}(m)\right)=0
$$

for $i>0$ and $m \gg 0$. For the same reasoning, the sheaves $\mathcal{L}_{\xi}$ satisfy the following base change property: let $\rho: T \rightarrow S$ be any base change and let $\rho^{*}(\xi) \in \mathcal{F}(T)$ be the pull back of $\xi$. Then there is a canonical isomorphism of sheaves of $\mathcal{O}_{T}$-modules

$$
\mathcal{L}_{\rho^{*}(\xi)} \cong\left(\mathbf{1}_{\mathbf{P}^{1}} \times \rho\right)^{*} \mathcal{L}_{\xi}
$$

Since $\mathcal{L}_{\xi}$ is flat over $S$, we can define the determinant line bundle of $\mathcal{L}_{\xi}$, denoted by $\operatorname{det}\left(\mathcal{L}_{\xi}\right)$ [28]. The sheaf $\operatorname{det}\left(\mathcal{L}_{\xi}\right)$ is an invertible sheaf over $\mathbf{P}^{1} \times S$. Using the Riemann-Roch theorem, one computes that its degree along fibers over $S$ are $d$. Further, because $\mathcal{L}_{\xi}$ has rank one, there is a canonical homomorphism

$$
\mathcal{L}_{\xi} \longrightarrow \operatorname{det}\left(\mathcal{L}_{\xi}\right)
$$

so that its kernel is the torsion subsheaf of $\mathcal{L}_{\xi}$, denoted by Tor $\left(\mathcal{L}_{\xi}\right)$. Let $w_{0}, \ldots, w_{n}$ be the homogeneous coordinate of $\mathbf{P}^{n}$ chosen before. $w_{0}, \ldots, w_{n}$ form a basis of $H^{0}\left(\mathbf{P}^{n}, \mathcal{O}_{\mathbf{P}^{n}}(1)\right)$. Then their pull backs provide a collection of canonical sections of $\mathcal{L}_{\xi}$, and hence a collection of canonical sections

$$
\sigma_{\xi, 0}, \ldots, \sigma_{\xi, n} \in H^{0}\left(S, \pi_{S *} \operatorname{det}\left(\mathcal{L}_{\xi}\right)\right)
$$


based on (2.3). Then after fixing an isomorphism

$$
\operatorname{det}\left(\mathcal{L}_{\xi}\right) \cong \pi_{S}^{*} \mathcal{M} \otimes \pi_{\mathbf{P}^{1}}^{*} \mathcal{O}_{\mathbf{P}^{1}}(d)
$$

for some invertible sheaf $\mathcal{M}$ of $\mathcal{O}_{S}$-modules, we obtain a section of

$$
\pi_{S^{*}}\left(\pi_{\mathbf{P}^{1}}^{*} \mathcal{O}_{\mathbf{P}^{1}}(d)\right) \otimes_{\mathcal{O}_{S}} \mathcal{M} \equiv H_{\mathbf{P}^{1}}^{0}\left(\mathcal{O}_{\mathbf{P}^{1}}(d)\right) \otimes_{\mathbf{C}} \mathcal{M}
$$

Finally, we let $w_{0}, w_{1}$ be the homogeneous coordinate of $\mathbf{P}^{1}$. Then the space $H_{\mathbf{P}^{1}}^{0}\left(\mathcal{O}_{\mathbf{P}^{1}}(d)\right)$ is the space of degree $d$ homogeneous polynomials in variables $w_{0}$ and $w_{1}$. This way, we obtain a morphism

$$
\Psi(S): S \longrightarrow N_{d}
$$

that is independent of the isomorphisms (2.4). It follows from the base change property (2.2) that the collection $\Psi(S)$ defines a transformation

$$
\Psi: \mathcal{F} \longrightarrow \operatorname{Mor}\left(-, N_{d}\right)
$$

thus defines a morphism $\varphi$ as desired.

It remains to check that for any $w \in S$, the sections

$$
\sigma_{\xi, 0}(w), \ldots, \sigma_{\xi, n}(w) \in H^{0}\left(\mathbf{P}^{1}, \operatorname{det}\left(\mathcal{L}_{\xi}\right) \otimes \mathcal{O}_{S} k_{w}\right)
$$

has the described vanishing property. Because of the base change property of $\mathcal{L}_{\xi}$, it suffices to check the statement when $S$ is a point and $\xi \in \mathcal{F}_{d}(S)$ is the stable map $f: C \rightarrow \mathbf{P}^{1} \times \mathbf{P}^{n}$. Let $x_{1}, \ldots, x_{N}$ be the set of points in $\mathbf{P}^{1}$ so that $p_{1}: C \rightarrow \mathbf{P}^{1}$, where $p_{1}=\pi_{\mathbf{P}^{1}} \circ f$, is not flat over these points. Let $C_{i}$ be $p_{1}^{-1}\left(x_{i}\right)$ and let $m_{i}$ be the degree of $f\left(\left[C_{i}\right]\right) \in H_{2}\left(\mathbf{P}^{n}\right)$. Then $\mathcal{L}_{\xi}=p_{1 *} p_{2}^{*} \mathcal{O}_{\mathbf{P}^{n}}(1)$ is locally free away from $x_{1}, \ldots, x_{N}$ and has torsion of length $m_{i}$ at $x_{i}$. Then $\mathcal{L}_{\xi} / \operatorname{Tor}\left(\mathcal{L}_{\xi}\right)$ is locally free of degree $k-\sum m_{i}$. It is direct to check that the canonical inclusion

$$
\mathcal{L}_{\xi} / \operatorname{Tor}\left(\mathcal{L}_{\xi}\right) \longrightarrow \operatorname{det}\left(\mathcal{L}_{\xi}\right) \cong \mathcal{O}_{\mathbf{P}^{1}}(d)
$$

has cokernel supported on the union of $x_{1}, \ldots, x_{N}$ whose length at $x_{i}$ is exactly $m_{i}$. The statement about the vanishing of $\sigma_{\xi, 0}(w), \ldots, \sigma_{\xi, n}(w)$ follows immediately.

The fact that $\varphi: M_{d} \rightarrow N_{d}$ is $S^{1} \times T$-equivariant as stated follows immediately from the fact that $\varphi$ is induced by the transformation $\Psi$ of functors. This completes the proof. 


\subsection{Concavex bundles}

Definition 2.7. We call a T-equivariant vector bundle $V \rightarrow \mathbf{P}^{n}$ convex (resp. concave) if the T-equivariant Euler class $e_{T}(V)$ is invertible and if $H^{1}\left(C, f^{*} V\right)=0$ (resp. $H^{0}\left(C, f^{*} V\right)=0$ ) for every 0-pointed genus 0 stable map $f: C \rightarrow \mathbf{P}^{n}$. We call $V$ a concavex bundle if it is a direct sum of a convex and a concave bundles. We denote by $V^{ \pm}$the convex and concave summands of $V$. By convention, we consider the zero bundle to be both convex and concave so that concavexity includes both convexity and concavity.

The convexity of a bundle is analogous to the notion of convexity of a projective manifold introduced by Behrend-Manin.

For example $\mathcal{O}(l) \rightarrow \mathbf{P}^{n}$ is convex if $l>0$, and concave if $l<0$. Given any concavex bundle $V \rightarrow \mathbf{P}^{n}$, we have a sequence of induced bundles

$$
U_{d} \rightarrow \overline{\mathcal{M}}_{0,0}\left(d, \mathbf{P}^{n}\right)
$$

whose fiber at $(f, C)$ is the space $H^{0}\left(C, f^{*} V^{+}\right) \oplus H^{1}\left(C, f^{*} V^{-}\right)$. Pulling back $U_{d}$ via the contracting map $\pi: M_{d} \rightarrow \overline{\mathcal{M}}_{0,0}\left(d, \mathbf{P}^{n}\right)$, we get a sequence of bundles

$$
V_{d}:=\pi^{*} U_{d} \rightarrow M_{d}
$$

We denote by $e_{T}\left(V_{d}\right)$ the equivariant Euler class of $V_{d}$. We also introduce the notations:

$$
\begin{aligned}
\Omega^{V} & :=\frac{e_{T}\left(V^{+}\right)}{e_{T}\left(V^{-}\right)} \\
Q_{d} & :=\varphi_{!} e_{T}\left(V_{d}\right), \quad Q_{0}:=\Omega^{V} .
\end{aligned}
$$

By convention, if $V$ is the zero bundle, we set $e_{T}\left(V_{d}\right)=1, e_{T}(V)=1$, $\Omega^{V}=1$.

Theorem 2.8. The sequence $\varphi_{!} e_{T}\left(V_{d}\right) \in H_{G}^{*}\left(N_{d}\right)$ is an $\Omega^{V}$-Euler data.

Proof: We first discuss some preliminaries. Let $M$ and $N$ be two compact smooth manifolds with the action of a torus $T$, and $\varphi$ : $M \rightarrow N$ be an equivariant map. Let $F$ be one component of the fixed submanifold in $N$ and $i_{F}$ be the inclusion map $F$ in $N$. Let $\phi_{F}=$ $i_{F !}(1) \in H_{T}^{*}(N)$ denote the equivariant Thom class of the normal bundle of $F$ in $N$. We then have, for any $\omega \in H_{T}^{*}(M)$ 


$$
\int_{M} \omega \varphi^{*}\left(\phi_{F}\right)=\int_{N} \varphi_{!}(\omega) \phi_{F}=\int_{F} i_{F}^{*}\left(\varphi_{!}(\omega)\right) .
$$

On the other hand, let $\{P\}$ be the components of the fixed submanifold contained in $\varphi^{-1}(F)$. By the Atiyah-Bott [7][3] localization formula on $M$, we get

$$
\sum_{P} \int_{P} i_{P}^{*}\left(\omega \varphi^{*}\left(\phi_{F}\right)\right) / e_{T}(P / M)=\int_{F} i_{F}^{*}\left(\varphi_{!}(\omega)\right) .
$$

Here $e_{T}(P / M)$ denotes the equivariant Euler class of the normal bundle of $P$ in $M$. The reason is that the contribution of the fixed point sets not contained in $\varphi^{-1}(F)$ is clearly zero. Actually if $Q$ is a component not contained inside $\varphi^{-1}(F)$, its contribution to the localization is given by

$$
\int_{Q} i_{Q}^{*}\left(\omega \varphi^{*}\left(\phi_{F}\right)\right) / e_{T}(Q / M)
$$

But by the naturality of the pull-backs, we have

$$
i_{Q}^{*} \varphi^{*}\left(\phi_{F}\right)=\varphi_{0}^{*} i_{E}^{*}\left(\phi_{F}\right)=0
$$

where $E=\varphi(Q)$ is a fixed submanifold in $N$ and $\varphi_{0}$ denotes the restriction of $\varphi$ to $Q$. Note that if $F$ is an isolated point, then $i_{P}^{*} \varphi^{*}\left(\phi_{F}\right)$ can be pulled out of the integral.

The above formula will be applied to the collapsing map $\varphi$ : $M_{d} \rightarrow N_{d}$. All manifolds involved here are at worst orbifolds with finite quotient singularities, so the localization formula remains valid without any change as long as we consider the corresponding integrals in the orbifold sense.

We consider the $S^{1}$-action on the $\mathbf{P}^{1}$ factor in $\mathbf{P}^{1} \times \mathbf{P}^{n}$ with weights $\alpha, 0$. Combining with the natural $T$-action on $\mathbf{P}^{n}$, we get the naturally induced $G=S^{1} \times T$-actions on $M_{d}$ and $N_{d}$, with respect to which the collapsing map $\varphi$ is equivariant. As described in section 2.1 , the $G$-fixed points in $N_{d}$ are all of the form

$$
p_{i r}=\left[0, \cdots, 0, w_{0}^{r} w_{1}^{d-r}, 0, \cdots, 0\right]
$$

in which the only nonzero term is in the $i$-th position.

For each $r>0$, let $\left\{F_{r}\right\} \subset \overline{\mathcal{M}}_{0,1}\left(r, \mathbf{P}^{n}\right)$ denote the $T$-fixed point components in $\overline{\mathcal{M}}_{0,1}\left(r, \mathbf{P}^{n}\right)$ with the marked point mapped onto the fixed point $p_{i}$ in $\mathbf{P}^{n}$. Let $N\left(F_{r}\right)=N_{F_{r} / \overline{\mathcal{M}}_{0,1}\left(r, \mathbf{P}^{n}\right)}$ denote the normal bundle of $F_{r}$ in $\overline{\mathcal{M}}_{0,1}\left(r, \mathbf{P}^{n}\right)$. 
Let $\pi_{1}, \pi_{2}$ be the projections from $\mathbf{P}^{1} \times \mathbf{P}^{n}$ onto the first and second factors. From the construction of $\varphi$, we see that the $G$-invariant submanifold that is mapped to $p_{i r}$ consists of the following degree $(1, d)$ stable maps $f: C \rightarrow \mathbf{P}^{1} \times \mathbf{P}^{n}$ with $C=C_{1} \cup C_{0} \cup C_{2}$. Here $C_{0} \simeq \mathbf{P}^{1}$ and

$$
\pi_{2} \circ f\left(C_{0}\right)=[0, \cdots, 0,1,0 \cdots, 0]=p_{i} \in \mathbf{P}^{n}
$$

where 1 is at the $i$-th position. The map $\pi_{1} \circ f: C_{0} \rightarrow \mathbf{P}^{1}$ is an isomorphism and maps $x_{1}=C_{0} \cap C_{1}$ and $x_{2}=C_{0} \cap C_{2}$ to 0 and $\infty$ respectively. Actually

$$
\pi_{1} \circ f\left(C_{1}\right)=0, \quad \pi_{1} \circ f\left(C_{2}\right)=\infty \text { in } \mathbf{P}^{1},
$$

ie. the curves $C_{1}$ and $C_{2}$ are respectively mapped to the points 0 and $\infty$ of $\mathbf{P}^{1}$.

The maps $\pi_{2} \circ f$ restricted to $C_{j}$ for $j=1,2$ are stable maps in $\overline{\mathcal{M}}_{0,1}\left(r, \mathbf{P}^{n}\right)$ and $\overline{\mathcal{M}}_{0,1}\left(d-r, \mathbf{P}^{n}\right)$ respectively. We consider $F_{r} \times F_{d-r}$ as a $G$-fixed submanifold of $M_{d}$ by gluing each pair to $C_{0}$ at $x_{1}$ and $x_{2}$ respectively as above. It is easy to see that $\left\{F_{r} \times F_{d-r}\right\}$ are the $G$-fixed point sets in $M_{d}$ whose image under $\varphi$ is the fixed point $p_{i r}$.

We first consider a convex bundle $V$ on $\mathrm{P}^{n}$, and the case $r \neq 0, d$. Then we have

$$
Q_{d}\left(\lambda_{i}+r \alpha\right)=\int_{N_{d}} \phi_{p_{i r}} Q_{d}=\int_{M_{d}} \varphi^{*}\left(\phi_{p_{i r}}\right) e_{T}\left(V_{d}\right)
$$

Here $\phi_{p_{\text {ir }}}$ denote the equivariant Thom class of the $G$-fixed point $p_{i r}$ in $N_{d}$. We will apply the localization formula to compute the right hand side of (2.5). First we need to know the normal bundle of the fixed points, which is, in the equivariant $K$-group of $F_{r} \times F_{d-r}$ [34][20],

$$
\begin{aligned}
N_{F_{r} \times F_{d-r} / M_{d}} & =N\left(F_{r}\right)+N\left(F_{d-r}\right)+\left[H^{0}\left(C_{0},\left(\pi_{1} \circ f\right)^{*} T \mathbf{P}^{1}\right)\right] \\
& +\left[L_{r} \otimes T_{x_{1}} C_{0}\right]+\left[L_{d-r} \otimes T_{x_{2}} C_{0}\right]-\left[T_{p_{i}} P^{n}\right]-\left[A_{C_{0}}\right] .
\end{aligned}
$$

Here $L_{r}$ denotes the line bundle on $\overline{\mathcal{M}}_{0,1}\left(r, \mathbf{P}^{n}\right)$ whose fiber at $\left(f_{1}, C_{1}, x_{1}\right)$ is the tangent line at $x_{1}$. Likewise for $L_{d-r}$. Their contributions correspond to the deformation of the nodal points $x_{1}$ and $x_{2}$. The term $H^{0}\left(C_{0},\left(\pi_{1} \circ f\right)^{*} T \mathbf{P}^{1}\right)$ corresponds to the deformation of $f$ restricted to $C_{0}$. The term $\left[A_{C_{0}}\right]$ is the bundle representing the infinitesimal automorphism of $C_{0}$ fixing the two points $x_{1}, x_{2}$. The term $-\left[T_{p_{i}} P^{n}\right]$ comes from gluing $F_{r}$ and $F_{d-r}$ onto $C_{0}$ and the property that $\pi_{2} \circ f\left(C_{0}\right)=p_{i}$. 
This gives the following formula for the corresponding equivariant Euler classes:

$$
\begin{aligned}
e_{T}\left(F_{r} \times F_{d-r} / M_{d}\right) & =e_{T}\left(N\left(F_{r}\right)\right) e_{T}\left(N\left(F_{d-r}\right)\right) e_{T}\left(L_{r} \otimes T_{x_{1}} C_{0}\right) \\
& \times e_{T}\left(L_{d-r} \otimes T_{x_{2}} C_{0}\right) e_{T}\left(T_{p_{i}} \mathbf{P}^{n}\right)^{-1} \\
& \times e_{T}\left(H^{0}\left(C_{0},\left(\pi_{1} \circ f\right)^{*} T \mathbf{P}^{1}\right)\right) e_{T}^{-1}\left(A_{C_{0}}\right)
\end{aligned}
$$

Each term in this formula can be explicitly calculated. We clearly have $e_{T}\left(T_{p_{i}} \mathbf{P}^{n}\right)=\prod_{j \neq i}\left(\lambda_{i}-\lambda_{j}\right)$; the weights of $T_{x_{1}} C_{0}$ and $T_{x_{2}} C_{0}$ are $\alpha$ and $-\alpha$ respectively, therefore

$e_{T}\left(L_{r} \otimes T_{x_{1}} C_{0}\right)=\alpha+c_{1}\left(L_{r}\right), \quad e_{T}\left(L_{d-r} \otimes T_{x_{2}} C_{0}\right)=-\alpha+c_{1}\left(L_{d-r}\right)$

where $c_{1}\left(L_{r}\right), c_{1}\left(L_{d-r}\right)$ are the restriction to $F_{r}$ and $F_{d-r}$ of the equivariant Chern classes of the line bundles $L_{r}$ and $L_{d-r}$ with respect to the induced $T$ actions on $\overline{\mathcal{M}}_{0,1}\left(r, \mathbf{P}^{n}\right)$ and $\overline{\mathcal{M}}_{0,1}\left(d-r, \mathbf{P}^{n}\right)$. To compute $e_{T}\left(H^{0}\left(C_{0},\left(\pi_{1} \circ f\right)^{*} T \mathbf{P}^{1}\right)\right)$ and $e_{T}\left(A_{C_{0}}\right)$, first note that we have the standard exact sequence

$$
0 \rightarrow O \rightarrow \mathcal{O}(1) \otimes \mathbf{C}^{2} \rightarrow T \mathbf{P}^{1} \rightarrow 0,
$$

with $O$ being the trivial bundle. From this we get

$$
0 \rightarrow O \rightarrow H^{0}\left(C_{0}, \mathcal{O}(1)\right) \otimes \mathbf{C}^{2} \rightarrow H^{0}\left(C_{0},\left(\pi_{1} \circ f\right)^{*}\left(T \mathbf{P}^{1}\right)\right) \rightarrow 0 .
$$

The weights of $H^{0}\left(C_{0}, \mathcal{O}(1)\right)$ are $\alpha, 0$, the weights of $\mathbf{C}^{2}$ are $-\alpha, 0$ and the weight of $O$ is 0 . Therefore one finds that the weights of $H^{0}\left(C_{0},\left(\pi_{1} \circ f\right)^{*}\left(T \mathbf{P}^{1}\right)\right)$ are $\alpha,-\alpha, 0$.

For $\left[A_{C_{0}}\right]$, we have the exact sequence

$$
0 \rightarrow A_{C_{0}} \rightarrow H^{0}\left(C_{0},\left(\pi_{1} \circ f\right)^{*}\left(T \mathbf{P}^{1}\right)\right) \rightarrow T_{x_{1}} C_{0} \oplus T_{x_{2}} C_{0} \rightarrow 0 .
$$

The weights of $T_{x_{1}} C_{0}$ and $T_{x_{2}} C_{0}$ are $\alpha$ and $-\alpha$ respectively. So $\left[A_{C_{0}}\right]$ contributes a 0 weight space which cancels with the 0 weight space of $\left[H^{0}\left(C_{0},\left(\pi_{1} \circ f\right)^{*}\left(T \mathbf{P}^{1}\right)\right)\right]$. Therefore we will ignore the zero weights in our formulas and write as

$$
e_{T}\left(H^{0}\left(C_{0},\left(\pi_{1} \circ f\right)^{*}\left(T \mathbf{P}^{1}\right)\right) e_{T}^{-1}\left(A_{C_{0}}\right)=-\alpha^{2} .\right.
$$


When $V_{d}$ is restricted to $F_{r} \times F_{d-r}$ considered as a fixed point set of $M_{d}$ as before, we have the exact sequence:

$$
\left.\left.\left.0 \rightarrow V_{d} \rightarrow V_{r}\right|_{F_{r}} \oplus V_{d-r}\right|_{F_{d-r}} \rightarrow V\right|_{p_{i}} \rightarrow 0
$$

Note that $\left.V_{r}\right|_{F_{r}}$ and $\left.V_{d-r}\right|_{F_{d-r}}$ is the same as $\left.\rho^{*} U_{r}\right|_{F_{r}}$ and $\left.\rho^{*} U_{d-r}\right|_{F_{d-r}}$ which respectively are the restrictions to $F_{r}$ and $F_{d-r}$ of the pull-backs to $\overline{\mathcal{M}}_{0,1}\left(r, \mathbf{P}^{n}\right)$ and $\overline{\mathcal{M}}_{0,1}\left(d-r, \mathbf{P}^{n}\right)$ of the corresponding bundles on $\overline{\mathcal{M}}_{0,0}\left(r, \mathbf{P}^{n}\right)$ and $\overline{\mathcal{M}}_{0,0}\left(d-r, \mathbf{P}^{n}\right)$. Here $\left.V\right|_{p_{i}}$ denotes the fiber of $V$ at $p_{i} \in \mathbf{P}^{n}$.

Here comes the important point. The multiplicativity of equivariant Euler classes gives us

$$
\Omega^{V}\left(\lambda_{i}\right) \cdot e_{T}\left(V_{d}\right)=e_{T}\left(V_{r}\right) \cdot e_{T}\left(V_{d-r}\right)=\rho^{*} e_{T}\left(U_{r}\right) \cdot \rho^{*} e_{T}\left(U_{d-r}\right)
$$

when restricted to $F_{r} \times F_{d-r} \subset M_{d}$. Here $\rho: \overline{\mathcal{M}}_{0,1}\left(d, \mathbf{P}^{n}\right) \rightarrow$ $\overline{\mathcal{M}}_{0,0}\left(d, \mathrm{P}^{n}\right)$ is the forgetting map (same notation for all $d$ ). Note that the above equality is just the pull-back via $\pi$ from $\overline{\mathcal{M}}_{0,0}\left(d, \mathbf{P}^{n}\right)$ of the gluing identity discussed in the Introduction.

For the case of $r=0$ or $d$, there is only one of the curves $C_{1}$ or $C_{2}$, ie. $C$ is of the form $C_{0} \cup C_{2}$ or $C_{1} \cup C_{0}$. In this case we identify $F_{d}$ as the fixed point set in $M_{d}$ by gluing its marked point to $C_{0}$ at $x_{1}$ or $x_{2}$. The normal bundle in these two cases are respectively given by

$$
N_{F_{d} / M_{d}}=N\left(F_{d}\right)+\left[H^{0}\left(C_{0},\left(\pi_{1} \circ f\right)^{*} T \mathbf{P}^{1}\right)\right]+\left[L_{d} \otimes T_{x_{j}} C_{0}\right]-\left[A_{C_{0}}\right]
$$

in the $K$-group of $F_{d}$. Here $L_{d}$ is the restriction to $F_{d}$ of the line bundle on $\overline{\mathcal{M}}_{0,1}\left(d, \mathbf{P}^{n}\right)$ whose fiber is the tangent line at the marked point. For simplicity we write $L$ as $L_{d}$ in the following. For $j=1,2$, $T_{x_{j}} C_{0}$ is the tangent line of $C_{0}$ at the corresponding marked point $x_{j}$. In these two cases, one easily shows in the same way as above that the term $e_{T}\left(A_{C_{0}}\right)$, except the 0 weight, contributes one nonzero weight $-\alpha$ or $\alpha$ respectively. Its 0 weight space still cancels with the 0 weight space of $\left[H^{0}\left(C_{0},\left(\pi_{1} \circ f\right)^{*} T \mathbf{P}^{1}\right)\right]$.

By putting all of the above computations together and combining 
with (2.5), we get, for $r \neq 0, d$,

$$
\begin{aligned}
\Omega^{V}\left(\lambda_{i}\right) Q_{d}\left(\lambda_{i}+r \alpha\right) & =\Omega^{V}\left(\lambda_{i}\right) \int_{M_{d}} \varphi^{*}\left(\phi_{p_{i r}}\right) e_{T}\left(V_{d}\right) \\
& =-\alpha^{-2} \prod_{j \neq i}\left(\lambda_{i}-\lambda_{j}\right) e_{T}\left(p_{i r} / N_{d}\right) \\
& \times \sum_{F_{r}} \int_{F_{r}} \frac{\rho^{*} e_{T}\left(U_{r}\right)}{e_{T}\left(N\left(F_{r}\right)\right)\left(\alpha+c_{1}\left(L_{r}\right)\right)} \\
& \times \sum_{F_{d-r}} \int_{F_{d-r}} \frac{\rho^{*} e_{T}\left(U_{d-r}\right)}{e_{T}\left(N\left(F_{d-r}\right)\right)\left(-\alpha+c_{1}\left(L_{d-r}\right)\right)}
\end{aligned}
$$

Here $e_{T}\left(p_{i r} / N_{d}\right)=\iota_{p_{i r}}^{*}\left(\phi_{p_{i r}}\right)$. Note that $\varphi^{*}\left(\phi_{p_{i r}}\right)$ restricted to $F_{r} \times$ $F_{d-r}$ is the same as $e_{T}\left(p_{i r} / N_{d}\right)$ which is a polynomial only in $\alpha$ and $\lambda$ as given below. Similarly for $r=d$, we have

$$
Q_{d}\left(\lambda_{i}+d \alpha\right)=\alpha^{-1} e_{T}\left(p_{i d} / N_{d}\right) \sum_{F_{d}} \int_{F_{d}} \frac{\rho^{*} e_{T}\left(U_{d}\right)}{e_{T}\left(N\left(F_{d}\right)\right)\left(\alpha+c_{1}(L)\right)}
$$

and for $r=0$

$$
Q_{d}\left(\lambda_{i}\right)=-\alpha^{-1} e_{T}\left(p_{i 0} / N_{d}\right) \sum_{F_{d}} \int_{F_{d}} \frac{\rho^{*} e_{T}\left(U_{d}\right)}{e_{T}\left(N\left(F_{d}\right)\right)\left(-\alpha+c_{1}(L)\right)}
$$

We can easily compute $e_{T}\left(p_{i r} / N_{d}\right)$ :

$$
e_{T}\left(p_{i r} / N_{d}\right)=\prod_{j=0}^{n} \prod_{m=0}^{d}\left(\lambda_{i}-\lambda_{j}+(r-m) \alpha\right) .
$$

For $r=0$ and $d$ we have

$$
\begin{aligned}
e_{T}\left(p_{i 0} / N_{d}\right) & =\prod_{j=0}^{n} \prod_{m=0}^{d}\left(\lambda_{i}-\lambda_{j}-m \alpha\right) \\
e_{T}\left(p_{i d} / N_{d}\right) & =\prod_{j=0}^{n} \prod_{m=0}^{d}\left(\lambda_{i}-\lambda_{j}+(d-m) \alpha\right) \\
& =\prod_{j=0}^{n} \prod_{m=0}^{d}\left(\lambda_{i}-\lambda_{j}+m \alpha\right) .
\end{aligned}
$$


The last two identities together with (2.7), (2.8) clearly gives us

$$
Q_{d}\left(\lambda_{i}+d \alpha\right)=\overline{Q_{d}\left(\lambda_{i}\right)}
$$

where $\bar{\alpha}=-\alpha, \bar{\lambda}_{i}=\lambda_{i}$. Finally our asserted quadratic relation:

$$
\Omega^{V}\left(\lambda_{i}\right) Q_{d}\left(\lambda_{i}+r \alpha\right)=\overline{Q_{r}\left(\lambda_{i}\right)} Q_{d-r}\left(\lambda_{i}\right)
$$

follows from (2.6), (2.7), (2.8), (2.9), and the following elementary identity:

$$
\begin{aligned}
& \prod_{j=0}^{n}\left(\lambda_{i}-\lambda_{j}\right) \times \prod_{j=0}^{n} \prod_{m=0}^{d}{ }_{(j, m) \neq(i, r)}\left(\lambda_{i}-\lambda_{j}+(r-m) \alpha\right) \\
& =\prod_{j=0}^{n} \prod_{m=0}^{r}\left(\lambda_{i}-\lambda_{j}+m \alpha\right) \\
& \quad \times \prod_{j=0}^{n} \prod_{m=0}^{d-r} \underset{(j, m) \neq(i, 0)}{\left(\lambda_{i}-\lambda_{j}-m \alpha\right)}
\end{aligned}
$$

Note that the last identity is just the interesting identity

$$
e_{T}\left(T_{p_{i}} \mathbf{P}^{n}\right) \cdot e_{T}\left(p_{i r} / N_{d}\right)=e_{T}\left(p_{i r} / N_{r}\right) \cdot e_{T}\left(p_{i 0} / N_{d-r}\right)
$$

When $V$ is concave, the fiber at $(f, C) \in \overline{\mathcal{M}}_{0,0}\left(d, \mathbf{P}^{n}\right)$ of the bundle $U_{d}$ is $H^{1}\left(C, f^{*} V\right)$, and we need only one change in the above argument. The gluing exact sequence in the concave case is

$$
\left.\left.\left.0 \rightarrow V\right|_{p_{i}} \rightarrow V_{d} \rightarrow V_{r}\right|_{F_{r}} \oplus V_{d-r}\right|_{F_{d-r}} \rightarrow 0
$$

instead. Therefore the gluing identity for equivariant Euler classes becomes

$$
e_{T}\left(V_{d}\right)=e_{T}\left(V_{r}\right) \cdot e_{T}\left(V_{d-r}\right) \iota_{p_{i}}^{*} e_{T}(V)
$$

Since $\Omega^{V}=1 / e_{T}(V)$ for concave $V$, the quadratic relation (2.10) remains valid in this case.

The case when $V$ is a direct sum of a convex and a concave bundle is also similar. 


\subsection{Linked Euler data}

Definition 2.9. Two sequences $P, Q \in \mathcal{S}$ are said to be linked if $\iota_{p_{i, 0}}^{*}\left(P_{d}-Q_{d}\right) \in \mathcal{R}^{-1}$ vanish at $\alpha=\left(\lambda_{i}-\lambda_{j}\right) / d$ for all $j \neq i, d>0$.

Let $V$ be a $T$-equivariant concavex bundle on $\mathbf{P}^{n}$, and $C \cong \mathbf{P}^{1}$ be any $T$-invariant line in $\mathbf{P}^{n}$. By Grothendieck's principle, we have the form

$$
\left.V\right|_{C}=\oplus_{a=1}^{N^{+}} \mathcal{O}\left(l_{a}\right) \oplus \oplus_{b=1}^{N^{-}} \mathcal{O}\left(-k_{b}\right)
$$

for some positive integers $l_{a}, k_{b}$. ( 0 cannot occurs because $e_{T}(V)$ is invertible, by definitiion.) Assume that $\left\{l_{a}\right\}$ and $\left\{k_{b}\right\}$ are independent of $C$. This is the case, for example, if $V$ is uniform [41]. We call the numbers $\left(l_{1}, . ., l_{N^{+}} ; k_{1}, . ., k_{N^{-}}\right)$the splitting type of $V$. With this notations, we have

Theorem 2.10. Let $Q_{d}=\varphi_{!} e_{T}\left(V_{d}\right)$ as before. At $\alpha=\left(\lambda_{j}-\lambda_{i}\right) / d$, $i \neq j$, we have

$$
\iota_{p_{j, 0}}^{*}\left(Q_{d}\right)=\prod_{a} \prod_{m=0}^{l_{a} d}\left(l_{a} \lambda_{j}-m\left(\lambda_{j}-\lambda_{i}\right) / d\right) \times \prod_{b} \prod_{m=1}^{k_{b} d-1}\left(-k_{a} \lambda_{j}+m\left(\lambda_{j}-\lambda_{i}\right) / d\right) .
$$

In particular the Euler data $Q$ is linked to

$$
P: \quad P_{d}=\prod_{a} \prod_{m=0}^{l_{a} d}\left(l_{a} \kappa-m \alpha\right) \times \prod_{b} \prod_{m=1}^{k_{b} d-1}\left(-k_{b} \kappa+m \alpha\right) .
$$

Proof: Since we shall evaluate the class $\iota_{p_{j, 0}}^{*}\left(Q_{d}\right)$ at $\alpha=\left(\lambda_{j}-\lambda_{i}\right) / d$, we introduce the notation $Q_{d}(\kappa, \alpha)=Q_{d}$, and denote the value of the class above by $Q_{d}\left(\lambda_{j},\left(\lambda_{j}-\lambda_{i}\right) / d\right)$. First consider the case $V=\mathcal{O}(l)$ on $\mathbf{P}^{n}$. We consider a smooth point in $(f, C) \in M_{d}$ with $C=\mathbf{P}^{1}$ and in coordinates

$$
f: C \rightarrow \mathbf{P}^{1} \times \mathbf{P}^{n}, \quad\left[w_{0}, w_{1}\right] \mapsto\left[w_{1}, w_{0}\right] \times\left[0, \cdots, w_{0}^{d}, \cdots, w_{1}^{d}, \cdots, 0\right] .
$$

where in the last term, $w_{0}^{d}$ is in the $i$-th position, $w_{1}^{d}$ is in the $j$-th position, and all of the other components are 0 . The image of $(f, C)$ in $N_{d}$ under $\varphi$ is the smooth point

$$
P_{i j}=\left[0, \cdots, w_{0}^{d}, \cdots, w_{1}^{d}, \cdots, 0\right] .
$$


It is easy to see that, if the weight of $S^{1}$ in the group $G=S^{1} \times T$ is $\alpha=\left(\lambda_{j}-\lambda_{i}\right) / d$, then $P_{i j}$ is fixed by the action of the subgroup of $G$ with $\alpha=\left(\lambda_{j}-\lambda_{i}\right) / d$. So $(f, C)$ is a smooth point in $M_{d}$ fixed by the subgroup in $G=S^{1} \times T$ with $\alpha=\left(\lambda_{j}-\lambda_{i}\right) / d$. The class $Q_{d}(\kappa, \alpha)$ restricted to $P_{i j}$ is just $Q_{d}\left(\lambda_{j},\left(\lambda_{j}-\lambda_{i}\right) / d\right)$.

At the points $(f, C) \in M_{d}$ and $P_{i j} \in N_{d}$, the map $\varphi$ is a canonical identification. From definition, $Q_{d}(\kappa, \alpha)$ restricted to $P_{i j}$ is the same as $e_{T}\left(V_{d}\right)$ restricted to $\varphi^{-1}\left(P_{i j}\right)=(f, C) \in M_{d}$, which by definition, is the same as $e_{T}\left(U_{d}\right)$ restricted to the $T$-fixed point $\left(\pi_{2} \circ f, C\right)$ in $\overline{\mathcal{M}}_{0,0}\left(d, \mathbf{P}^{n}\right)$. Note that $\left(\pi_{2} \circ f, C\right)$ is the degree $d$ cover of the $T$ invariant line joining $p_{i}$ and $p_{j}$ in $\mathbf{P}^{n}$. Explicitly

$$
\pi_{2} \circ f:\left[w_{0}, w_{1}\right] \rightarrow\left[0, \cdots, w_{0}^{d}, \cdots, w_{1}^{d}, \cdots 0\right] .
$$

So the induced action of $T$ on $C=\mathbf{P}^{1}$ has weights $\left\{\lambda_{i} / d, \lambda_{j} / d\right\}$.

Now let us compute $e_{T}\left(U_{d}\right)$ restricted to $\left(\pi_{2} \circ f, C\right)$. When restricted $\left(\pi_{2} \circ f, C\right) \in \overline{\mathcal{M}}_{0,0}\left(d, \mathbf{P}^{n}\right)$ the fiber of $U_{d}$ is just the section space $H^{0}\left(C,\left(\pi_{2} \circ f\right)^{*} \mathcal{O}(l)\right)=H^{0}(C, \mathcal{O}(l d))$. It has an explicit basis $\left\{w_{0}^{k} w_{1}^{l d-k}\right\}$ with $k=0, \cdots, l d$. Since the $T$-weight of $w_{0}^{k} w_{1}^{l d-k}$ is $k \lambda_{i} / d+(l d-k) \lambda_{j} / d$, by multiplying them together we get

$$
Q_{d}\left(\lambda_{j},\left(\lambda_{j}-\lambda_{i}\right) / d\right)=\prod_{m=0}^{l d}\left(l \lambda_{j}-m\left(\lambda_{j}-\lambda_{i}\right) / d\right) .
$$

For a general convex vector bundle $V$ on $\mathbf{P}^{n}$, the fiber of the induced bundle $V_{d}$ restricted to $\varphi^{-1}\left(P_{i j}\right)=(f, C) \in M_{d}$ is $H^{0}\left(\mathbf{P}^{1},\left(\pi_{2} \circ f\right)^{*} V\right)$. By Grothendieck's principle, $V$ restricted to the line spanning $p_{i}, p_{j}$ splits into direct sum of line bundles $\left\{\mathcal{O}\left(l_{a}\right)\right\}$. Pulling them back to $C$ via the degree $d$ map $\pi_{2} \circ f$, we get the direct sum of $\left\{\mathcal{O}\left(l_{a} d\right)\right\}$. Since $V$ is convex, each $l_{a}>0$. By applying the same computation to each summand, we get

$$
Q_{d}\left(\lambda_{j},\left(\lambda_{j}-\lambda_{i}\right) / d\right)=\prod_{a} \prod_{m=0}^{l_{a} d}\left(l_{a} \lambda_{j}-m\left(\lambda_{j}-\lambda_{i}\right) / d\right) .
$$

For a concave bundle $V$, we need only one minor change in the above argument. We leave to the reader as an exercise to check that for $V=\mathcal{O}(-k), k>0$,

$$
Q_{d}\left(\lambda_{j},\left(\lambda_{j}-\lambda_{i}\right) / d\right)=\prod_{m=1}^{k d-1}\left(-k \lambda_{j}+m\left(\lambda_{j}-\lambda_{i}\right) / d\right)
$$


by using either the Atiyah-Bott fixed point formula or by writing down an explicit basis for $H^{1}\left(\mathbf{P}^{1}, \mathcal{O}(-k d)\right)$. So for an arbitrary concave bundle $V$, we have the form

$$
Q_{d}\left(\lambda_{j},\left(\lambda_{j}-\lambda_{i}\right) / d\right)=\prod_{b} \prod_{m=1}^{k_{b} d-1}\left(-k_{b} \lambda_{j}+m\left(\lambda_{j}-\lambda_{i}\right) / d\right) .
$$

Similarly for an arbitrary concavex bundle $V$, we have the form

$$
\begin{aligned}
Q_{d}\left(\lambda_{j},\left(\lambda_{j}-\lambda_{i}\right) / d\right)= & \prod_{a} \prod_{m=0}^{l_{a} d}\left(l_{a} \lambda_{j}-m\left(\lambda_{j}-\lambda_{i}\right) / d\right) \\
& \times \prod_{b} \prod_{m=1}^{k_{b} d-1}\left(-k_{b} \lambda_{j}+m\left(\lambda_{j}-\lambda_{i}\right) / d\right) .
\end{aligned}
$$

Theorem 2.11. Suppose $P, Q$ are any linked $\Omega$-Euler data. If

$$
\operatorname{deg}_{\alpha} \iota_{p_{i, 0}}^{*}\left(P_{d}-Q_{d}\right) \leq(n+1) d-2
$$

for all $i=0, . ., n$ and $d=1,2, . .$, then $P=Q$.

Proof: By definition, $P_{0}=Q_{0}=\Omega$. We will show that $P_{d}=Q_{d}$, assuming that

$$
P_{r}=Q_{r}, \quad r=0, . ., d-1 .
$$

Since the $\mathcal{R}$-valued pairing $p f_{d}(u \cdot v)$ on $\mathcal{R} H_{G}^{*}\left(N_{d}\right)$ is nondegenerate, it suffices to show that

$$
L_{s}:=p f_{d}\left(\kappa^{s} \cdot\left(P_{d}-Q_{d}\right)\right)
$$

is zero for all $s=0,1,2, \ldots$. By the localization formula for $p f_{d}$, we get

$$
L_{s}=\sum_{i=0}^{n} \sum_{r=0}^{d}\left(\lambda_{i}+r \alpha\right)^{s} \frac{\iota_{p_{i r}}^{*}\left(P_{d}-Q_{d}\right)}{\prod_{k=0}^{n} \prod_{m=0(k, m) \neq(i, r)}^{d}\left(\lambda_{i}-\lambda_{k}+(r-m) \alpha\right)} .
$$

Since $P$ is an Euler data, it follows that $\iota_{p_{i r}}^{*}\left(P_{d}\right)$, for each $r=$ $1, . ., d-1$, is expressible in terms of $P_{1}, . ., P_{d-1}$. Likewise for $Q$. Thus 
by the inductive hypothesis (2.11), the sum over $r$ above receives contributions only from the $r=0, d$ terms. Applying the Reciprocity Lemma (i), we further simplify $L_{s}$ to

$$
\begin{aligned}
L_{s} & =\sum_{i=0}^{n}\left(\frac{\lambda_{i}^{s} A_{i}(\alpha)}{\alpha^{d}}+\frac{\left(\lambda_{i}+d \alpha\right)^{s} A_{i}(-\alpha)}{(-\alpha)^{d}}\right) \\
A_{i}(\alpha) & :=\frac{(-1)^{d}}{d ! \prod_{k \neq i}\left(\lambda_{i}-\lambda_{k}\right)} \frac{\iota_{p_{i, 0}}^{*}\left(P_{d}-Q_{d}\right)}{\prod_{k \neq i} \prod_{m=1}^{d}\left(\lambda_{i}-\lambda_{k}-m \alpha\right)} .
\end{aligned}
$$

Since $P, Q$ are linked Euler data, we have

$$
\iota_{p_{i, 0}}^{*}\left(P_{d}-Q_{d}\right)=0
$$

at $\alpha=\left(\lambda_{i}-\lambda_{k}\right) / d, k \neq i$. By the inductive hypothesis (2.11) and the Reciprocity Lemma (iii), (2.13) holds at $\alpha=\left(\lambda_{i}-\lambda_{k}\right) / m$ for $m=1, . ., d$ as well. This shows that $A_{i} \in \mathcal{R}=\mathbf{Q}(\lambda)[\alpha]$ for all $i$. By assumption $\operatorname{deg}_{\alpha} A_{i}<(n+1) d-1-n d=d-1$. But since $L_{s} \in \mathcal{R}$ ie. polynomial in $\alpha$, for all $s$, it follows easily that the $A_{i}$ must be identically zero.

\subsection{The Lagrange map and mirror transformations}

Throughout this subsection, we fix an invertible class $\Omega$ and shall denote by $\mathcal{A}=\mathcal{A}^{\Omega}$ the set of $\Omega$-Euler data.

Definition 2.12. An invertible map $\mu: \mathcal{A} \rightarrow \mathcal{A}$ is called a mirror transformation if for any $P \in \mathcal{A}, \mu(P)$ is linked to $P$. We call $\mu(P)$ a mirror transform of $P$.

Definition 2.13. (Notations) $\mathcal{S}_{0}$ denotes the set of sequences $B: \quad B_{d} \in \mathcal{R}^{-1} H_{G}^{*}\left(N_{0}\right), \quad d=1,2, \ldots$ We define the map $\mathcal{I}: \mathcal{S} \rightarrow \mathcal{S}_{0}$, $P \mapsto \mathcal{I}(P)=B$ where $B_{d}=I_{d}^{*}\left(P_{d}\right)$.

Recall that any equivariant cohomology class $\omega \in \mathcal{R}^{-1} H_{G}^{*}\left(N_{d}\right)$ is determined by its restrictions $\iota_{p_{i r}}^{*}(\omega) \in \mathcal{R}^{-1}, i=0, . ., n, r=0, . ., d$. Conversely given any collection $\omega_{i r} \in \mathcal{R}^{-1}$, there exists a unique class $\omega \in \mathcal{R}^{-1} H_{G}^{*}\left(N_{d}\right)$ such that $\iota_{p_{i r}}^{*}(\omega)=\omega_{i r}$ for all $i, r$. In fact,

$$
\omega=\sum_{i=0}^{n} \sum_{r=0}^{d} \omega_{i r} \prod_{(j, m) \neq(i, r)} \frac{\kappa-\lambda_{j}-m \alpha}{\lambda_{i}-\lambda_{j}-(m-r) \alpha} .
$$


In particular given a sequence $B \in \mathcal{S}_{0}$, then for each $d$ there is a unique class $P_{d} \in \mathcal{R}^{-1} H_{G}^{*}\left(N_{d}\right)$ such that

$$
\iota_{p_{i r}}^{*}\left(P_{d}\right)=\iota_{p_{i}}^{*}(\Omega)^{-1} \overline{\iota_{p_{i}}^{*}\left(B_{r}\right)} \iota_{p_{i}}^{*}\left(B_{d-r}\right), \quad i=0, . ., n, \quad r=0, . ., d,
$$

where we have set $B_{0}:=\Omega$. This defines a sequence $P \in \mathcal{S}$, hence a map

$$
\mathcal{L}_{\Omega}: \mathcal{S}_{0} \rightarrow \mathcal{S}, \quad B \mapsto \mathcal{L}_{\Omega}(B)=P .
$$

We shall call $\mathcal{L}=\mathcal{L}_{\Omega}$ the Lagrange map. By (2.14) at $r=0$, we get

$$
\iota_{p_{i}}^{*}\left(B_{d}\right)=\iota_{p_{i, 0}}^{*}\left(P_{d}\right)=\iota_{p_{i}}^{*} I_{d}^{*}\left(P_{d}\right), \quad i=0, . ., n .
$$

First, this implies that the two classes $B_{d}, I_{d}^{*}\left(P_{d}\right)$ on $N_{0}=\mathbf{P}^{n}$ coincide for each $d$. Thus

$$
B=\mathcal{I}(P)=\mathcal{I} \circ \mathcal{L}(B)
$$

Thus $\mathcal{L}: \mathcal{S}_{0} \rightarrow \mathcal{S}$ is a section of the onto map $\mathcal{I}: \mathcal{S} \rightarrow \mathcal{S}_{0}$. Second, substituting (2.15) into (2.14), we get

$$
\iota_{p_{i}}^{*}(\Omega) \iota_{p_{i r}}^{*}\left(P_{d}\right)=\overline{\iota_{p_{i, 0}}^{*}\left(P_{r}\right)} \iota_{p_{i, 0}}^{*}\left(P_{d-r}\right)
$$

If, furthermore, we have $P_{d} \in \mathcal{R} H_{G}^{*}\left(N_{d}\right)$ rather than in $\mathcal{R}^{-1} H_{G}^{*}\left(N_{d}\right)$, then eqn. (2.17) says that $P$ is an Euler data.

(A) The image $P=\mathcal{L}(B)$ of a given $B \in \mathcal{S}_{0}$ under the Lagrange map is an Euler data if $P_{d} \in \mathcal{R} H_{G}^{*}\left(N_{d}\right), d>0$.

On the other hand, it is trivial to show that if $Q \in \mathcal{A} \subset \mathcal{S}$ then

$$
Q=\mathcal{L} \circ \mathcal{I}(Q)
$$

Now using $\mathcal{L}$ we can lift any map $\mu_{0}: \mathcal{S}_{0} \rightarrow \mathcal{S}_{0}$ to a map

$$
\mu=\mathcal{L} \circ \mu_{0} \circ \mathcal{I}: \mathcal{S} \rightarrow \mathcal{S},
$$

which we shall call the Lagrange lift of $\mu_{0}$. Thus from eqns. (2.16) and (2.18), we have

(B) Let $\mu_{0}: \mathcal{S}_{0} \rightarrow \mathcal{S}_{0}$ be invertible with inverse $\nu_{0}$, and let $\mu, \nu$ be their respective Lagrange lifts. Then $\mu \circ \nu=\nu \circ \mu=i d_{\mathcal{A}}$ when restricted to Euler data.

We now discuss the relationship between Euler data and series of hypergeometric type. 
Definition 2.14. Given any $B \in \mathcal{S}_{0}$, define

$$
H G[B](t):=e^{-p t / \alpha}\left(\Omega+\sum_{d>0} \frac{B_{d} e^{d t}}{\prod_{k=0}^{n} \prod_{m=1}^{d}\left(p-\lambda_{k}-m \alpha\right)}\right)
$$

where $p \in H_{G}^{*}\left(N_{0}\right)$ is the equivariant hyperplane class of $N_{0}=\mathbf{P}^{n}$.

Note that $H G[B](t)$ is a cohomology valued formal series. If $P: P_{d}=\prod_{m=0}^{(n+1) d}(l \kappa-m \alpha)$ as in Example 1, it is obvious that in the limit $\lambda \rightarrow 0$, we have

$$
H G[\mathcal{I}(P)](t)=e^{-H t / \alpha} \sum_{d \geq 0} \frac{\prod_{m=0}^{(n+1) d}((n+1) H-m \alpha)}{\prod_{m=1}^{d}(H-m \alpha)^{n+1}} e^{d t}
$$

where $H \in H^{*}\left(\mathbf{P}^{n}\right)$ on the right hand side is the hyperplane class of $\mathbf{P}^{n}$. The coefficients of $\left(-\frac{H}{\alpha}\right)^{i}$ for $i=1, \ldots, n$, are exactly solutions to a hypergeometric differential equation discussed in the Introduction.

We now consider a construction of mirror transformations. Let $B \in \mathcal{S}_{0}$, and set $B_{0}:=\Omega$. Given any power series $g \in e^{t} \mathcal{R}\left[\left[e^{t}\right]\right]$, there is a unique $\tilde{B} \in \mathcal{S}_{0}$ such that

$$
H G[B](t+g)=H G[\tilde{B}](t) .
$$

In fact, since

$$
H G[B](t+g)=e^{-p t / \alpha} e^{-p g / \alpha} \sum_{d \geq 0} \frac{B_{d} e^{d t} e^{d g}}{\prod_{k=0}^{n} \prod_{m=1}^{d}\left(p-\lambda_{k}-m \alpha\right)},
$$

if we write $e^{d g}=\sum_{s \geq 0} g_{d, s} e^{s t}, g_{d, s} \in \mathcal{R}$ and $e^{-p g / \alpha}=\sum_{s \geq 0} g_{s}^{\prime} e^{s t}$, $g_{s}^{\prime} \in \mathcal{R}[p / \alpha]$, then it is straightforward to find that

$$
\begin{aligned}
& \tilde{B}_{d}=B_{d}^{\prime}+\sum_{r=0}^{d-1} g_{d-r}^{\prime} B_{r}^{\prime} \prod_{j=0}^{n} \prod_{m=r+1}^{d}\left(p-\lambda_{j}-m \alpha\right) \\
& B_{d}^{\prime}:=B_{d}+\sum_{r=0}^{d-1} g_{r, d-r} B_{r} \prod_{j=0}^{n} \prod_{m=r+1}^{d}\left(p-\lambda_{j}-m \alpha\right) .
\end{aligned}
$$

Thus we have an invertible transformation $\mu_{0}: \mathcal{S}_{0} \rightarrow \mathcal{S}_{0}, B \mapsto \tilde{B}$. Similarly, given any power series $f \in e^{t} \mathcal{R}\left[\left[e^{t}\right]\right]$ we have an invertible transformation $\mu_{0}: \mathcal{S}_{0} \rightarrow \mathcal{S}_{0}, B \mapsto \tilde{B}$, such that

$$
e^{f / \alpha} H G[B](t)=H G[\tilde{B}](t) \text {. }
$$


Again if we write $e^{f / \alpha}=\sum_{s \geq 0} f_{s} e^{s t}, f_{s} \in \mathcal{R}\left[\alpha^{-1}\right]$, then

$$
\tilde{B}_{d}=B_{d}+\sum_{r=0}^{d-1} f_{d-r} B_{r} \prod_{j=0}^{n} \prod_{m=r+1}^{d}\left(p-\lambda_{j}-m \alpha\right) .
$$

We now make an important observation about the transformation $\mu_{0}$ in each case above. For $d>0$ the class $\prod_{j=0}^{n}\left(p-\lambda_{j}-d \alpha\right)$ always vanishes when restricted to the fixed points $p_{i} \in \mathbf{P}^{n}$, at $\alpha=\left(\lambda_{i}-\right.$ $\left.\lambda_{j}\right) / d$. It follows immediately from (2.19) and (2.20) that $\iota_{p_{i}}^{*}\left(B_{d}\right)$, $\iota_{p_{i}}^{*}\left(\tilde{B}_{d}\right)$ always agree (whenever defined for all $d$ ) at $\alpha=\left(\lambda_{i}-\lambda_{j}\right) / d$, for $j \neq i$. To summarize:

(C) Given $g, f \in e^{t} \mathcal{R}\left[\left[e^{t}\right]\right]$, let $\mu_{0}: \mathcal{S}_{0} \rightarrow \mathcal{S}_{0}, B \mapsto \tilde{B}$, be the invertible transformation defined by

$$
e^{f / \alpha} H G[B](t+g)=H G[\tilde{B}](t) .
$$

Suppose $B$ is such that all values of $\iota_{p_{i}}^{*}\left(B_{d}\right)$ are well-defined at $\alpha=\left(\lambda_{i}-\lambda_{j}\right) / d, j \neq i$. Then these values are preserved under $\mu_{0}$.

Obviously if $P$ is an Euler data and $B=\mathcal{I}(P)$, then the restrictions $\iota_{p_{i}}^{*}\left(B_{d}\right)=\iota_{p_{i, 0}}^{*}\left(P_{d}\right) \in \mathcal{R}$ are polynomial in $\alpha$. Hence they are always well-defined at $\alpha=\left(\lambda_{i}-\lambda_{j}\right) / d, j \neq i$.

Lemma 2.15. Let $\mu$ be the Lagrange lift of the above transformation $\mu_{0}: \mathcal{S}_{0} \rightarrow \mathcal{S}_{0}, B \mapsto \tilde{B}$. Then $\mu$ is a mirror transformation. In particular, if $P$ is an Euler data, then $\tilde{P}=\mu(P)$ is an Euler data with

$$
e^{f / \alpha} H G[\mathcal{I}(P)](t+g)=H G[\mathcal{I}(\tilde{P})](t) .
$$

Proof: The second assertion follows from the first assertion and the fact that $\mathcal{I} \circ \mu=\mathcal{I} \circ \mathcal{L} \circ \mu_{0} \circ \mathcal{I}=\mu_{0} \circ \mathcal{I}$.

It suffices to consider the two cases $f=0, g=0$, separately. In each case we let $P$ be an Euler data, and denote

$$
\tilde{P}=\mu(P), \quad B=\mathcal{I}(P), \quad \tilde{B}=\mu_{0}(B) .
$$

Since $\mu:=\mathcal{L} \circ \mu_{0} \circ \mathcal{I}$, we have $\tilde{P}=\mathcal{L}(\tilde{B})$. In each case we will show that $\tilde{P}$ is an Euler data. We claim that this suffices. First, by statement 
(B) above, $\mu$ is invertible as a transformation on the set $\mathcal{A}$ of Euler data. Second, by statement (C) above, the restrictions $\iota_{p_{i}}^{*}\left(B_{d}\right)=$ $\iota_{p_{i, 0}}^{*}\left(P_{d}\right)$ and $\iota_{p_{i}}^{*}\left(\tilde{B}_{d}\right)=\iota_{p_{i, 0}}^{*}\left(\tilde{P}_{d}\right)$ agree at $\alpha=\left(\lambda_{i}-\lambda_{j}\right) / d, j \neq i$. Thus $\tilde{P}$ is linked to $P$. So, by definition, $\mu$ is a mirror transformation. We now proceed to checking Eulerity of $\tilde{P}$. Since $P=\mathcal{L}(B)$, (2.14) holds. Multiply both sides of eqn. (2.14) by the respective sides of the following identity:

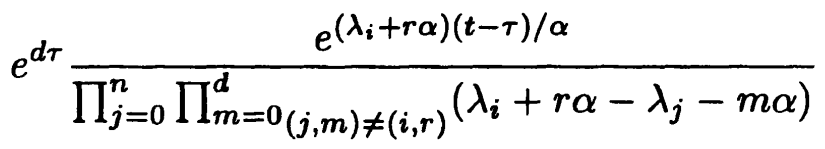

$$
\begin{aligned}
& =\frac{1}{\prod_{j \neq i}\left(\lambda_{i}-\lambda_{j}\right)} \times e^{\lambda_{i} t / \alpha} \frac{e^{r t}}{\prod_{j=0}^{n} \prod_{m=1}^{r}\left(\lambda_{i}-\lambda_{j}+m \alpha\right)} \\
& \times e^{-\lambda_{i} \tau / \alpha} \frac{e^{(d-r) \tau}}{\prod_{j=0}^{n} \prod_{m=1}^{d-r}\left(\lambda_{i}-\lambda_{j}-m \alpha\right)},
\end{aligned}
$$

and then sum over $i=0, . ., n$, and $r=0, . ., d$. The result is

$$
\begin{aligned}
& e^{d \tau} p f_{d}\left(P_{d} e^{\kappa(t-\tau) / \alpha}\right) \\
& =\sum_{r=0}^{d} p f\left(\Omega^{-1} \frac{B_{r} e^{r t}}{\left[e^{-p t / \alpha} \frac{B_{d-r} e^{(d-r) \tau}}{\prod_{j=0}^{n} \prod_{m=1}^{r}\left(p-\lambda_{j}-m \alpha\right)}\right]}\right. \\
& \times\left[e^{-p \tau / \alpha} \frac{\left.\prod_{j=0}^{n}\right]}{\prod_{m=1}^{d-r}\left(p-\lambda_{j}-m \alpha\right)} .\right.
\end{aligned}
$$

Now summing this over $d=0,1,2, .$. , we get:

$$
\sum_{d \geq 0} e^{d \tau} p f_{d}\left(P_{d} e^{\kappa(t-\tau) / \alpha}\right)=p f\left(\Omega^{-1} \overline{H G[B](t)} H G[B](\tau)\right) .
$$

Likewise, of course, for $\tilde{P}$ and $\tilde{B}$.

First case:

$$
H G[\tilde{B}](t)=H G[B]\left(t+g\left(e^{t}\right)\right)
$$

By (2.21), we have

$$
\begin{aligned}
& p f\left(\Omega^{-1} \overline{H G[\tilde{B}](t)} H G[\tilde{B}](\tau)\right)=\sum_{d \geq 0} e^{d \tau} p f_{d}\left(\tilde{P}_{d} e^{\kappa(t-\tau) / \alpha}\right) \\
& p f\left(\Omega^{-1} \overline{H G[B]\left(t+g\left(e^{t}\right)\right)} H G[B]\left(\tau+g\left(e^{\tau}\right)\right)\right) \\
& =\sum_{d \geq 0} e^{d\left(\tau+g\left(e^{\tau}\right)\right)} p f_{d}\left(P_{d} e^{\kappa\left(t+\bar{g}\left(e^{t}\right)-\tau-g\left(e^{\tau}\right)\right) / \alpha}\right) .
\end{aligned}
$$


By (2.22), we can equate the two right hand sides above. Setting $q=e^{\tau}, \zeta=(t-\tau) / \alpha$, we get

$$
\begin{gathered}
\sum_{d \geq 0} q^{d} p f_{d}\left(\tilde{P}_{d} e^{\zeta \kappa}\right)=\sum_{d \geq 0} q^{d} e^{d g(q)} p f_{d} \\
\times\left(P_{d} e^{\kappa \zeta} e^{\kappa\left(g_{+}\left(q e^{\zeta \alpha}\right)-g_{+}(q)\right) / \alpha} e^{-\kappa\left(g_{-}\left(q e^{\zeta \alpha}\right)+g_{-}(q)\right) / \alpha}\right)
\end{gathered}
$$

where $g=g_{+}+g_{-}$with $\bar{g}_{ \pm}= \pm g_{ \pm}$. Obviously for any $g(q) \in \mathcal{R}[[q]]$, $g_{+}\left(q e^{\zeta \alpha}\right)-g_{+}(q) \in \alpha \cdot \mathcal{R}[[q, \zeta]]$. Since the involution $\omega \mapsto \bar{\omega}$ on $\mathcal{R}$ simply changes the sign of $\alpha$, the fact that $g_{-}$is odd shows that $g_{-}(q) \in \alpha \cdot \mathcal{R}[[q]]$. Likewise for $g_{-}\left(q e^{\zeta \alpha}\right)$. We know that $P_{d} \in \mathcal{R} H_{G}^{*}\left(N_{d}\right)$ (since $P$ is Euler), and that $p f_{d}$ maps $\mathcal{R} H_{G}^{*}\left(N_{d}\right)$ to $\mathcal{R}$. So the right hand side of $(2.23)$ now clearly lies in $\mathcal{R}[[q, \zeta]]$. So, likewise for the left hand side of (2.23). It follows that

$$
p f_{d}\left(\tilde{P}_{d} \kappa^{s}\right) \in \mathcal{R}, \quad s=0,1,2, \ldots
$$

A priori $\tilde{P}_{d} \in \mathcal{R}^{-1} H_{G}^{*}\left(N_{d}\right)$ has the form

$$
\tilde{P}_{d}=a_{N} \kappa^{N}+\cdots+a_{0}, \quad a_{i} \in \mathcal{R}^{-1}, \quad N=(n+1) d+n .
$$

Since $p f_{d}\left(\kappa^{N}\right)=1$, it follows from (2.24) that $a_{N}, . ., a_{0} \in \mathcal{R}$. Hence $\tilde{P}_{d} \in \mathcal{R} H_{G}^{*}\left(N_{d}\right)$ (rather than in $\mathcal{R}^{-1} H_{G}^{*}\left(N_{d}\right)$ ). By statement (A) above, $\tilde{P}$ is an Euler data.

Second case:

$$
H G[\tilde{B}](t)=e^{f / \alpha} H G[B](t) .
$$

Again applying (2.21) and writing $f \in e^{t} \mathcal{R}\left[\left[e^{t}\right]\right]$ as $f=f_{+}+f_{-}$with $\bar{f}_{ \pm}= \pm f_{ \pm}$, we get

$$
\begin{aligned}
\sum_{d \geq 0} q^{d} p f_{d}\left(\tilde{P}_{d} e^{\zeta \kappa}\right) & =e^{-\bar{f}\left(e^{t}\right) / \alpha} e^{f\left(e^{\tau}\right) / \alpha} p f\left(\Omega^{-1} \overline{H G[B](t)} H G[B](\tau)\right) \\
& =e^{-\left(f_{+}\left(q e^{\zeta \kappa}\right)-f_{+}(q)\right) / \alpha} e^{\left(f_{-}\left(q e^{\zeta \kappa}\right)+f_{-}(q)\right) / \alpha} \\
& \times \sum_{d \geq 0} q^{d} p f_{d}\left(P_{d} e^{\zeta \kappa}\right)
\end{aligned}
$$

The right hand side lies in $\mathcal{R}[[q, \zeta]]$ as before, implying that $\tilde{P}$ is Euler. 


\section{Applications}

Definition 3.1. A concavex bundle $V$ on $\mathbf{P}^{n}$ is called a critical bundle if the induced bundle $U_{d} \rightarrow \overline{\mathcal{M}}_{0,0}\left(d, \mathbf{P}^{n}\right)$ has rank dim $\overline{\mathcal{M}}_{0,0}\left(d, \mathbf{P}^{n}\right)=$ $(n+1) d+n-3$. We denote the nonequivariant Euler class by $c_{t o p}\left(U_{d}\right)$. we have

Recall the notation that given a concavex bundle $V=V^{+} \oplus V^{-}$,

$$
\begin{aligned}
& \Omega^{V}:=e_{T}\left(V^{+}\right) / e_{T}\left(V^{-}\right) \\
& Q: \quad Q_{d}=\varphi_{!} e_{T}\left(V_{d}\right), \quad d>0 .
\end{aligned}
$$

By Theorem 2.8 the sequence $Q$ is an $\Omega^{V}$-Euler data. If $V$ is a critical bundle, introduce

$$
K_{d}:=\int_{\overline{\mathcal{M}}_{0,0}\left(d, \mathbf{P}^{n}\right)} c_{t o p}\left(U_{d}\right), \quad \Phi:=\sum_{d>0} K_{d} e^{d t} .
$$

Theorem 3.2. Let $V$ be a concavex bundle on $\mathrm{P}^{n}$.

(i) The restrictions $I_{d}^{*}\left(Q_{d}\right) \in H_{G}^{*}\left(\mathbf{P}^{n}\right)$ has $\operatorname{deg}_{\alpha} I_{d}^{*}\left(Q_{d}\right) \leq(n+1) d-2$.

(ii) If $V$ is critical, then in the nonequivariant limit $\lambda \rightarrow 0$,

$$
\begin{aligned}
& \int_{\mathbf{P}^{n}} e^{-H t / \alpha} \frac{I_{d}^{*}\left(Q_{d}\right)}{\prod_{m=1}^{d}(H-m \alpha)^{n+1}}=\alpha^{-3}(2-d t) K_{d} \\
& \int_{\mathbf{P}^{n}}\left(H G[\mathcal{I}(Q)](t)-e^{-H t / \alpha} \Omega^{V}\right)=\alpha^{-3}\left(2 \Phi-t \Phi^{\prime}\right) .
\end{aligned}
$$

Proof: The second equality in (ii) follows trivially from the first equality.

By eqn. (2.8) in the proof of Theorem 2.8, we have

$$
Q_{d}\left(\lambda_{i}\right)=\phi_{p_{i}}\left(\lambda_{i}\right) \phi_{\mathbf{P}^{n}}\left(\lambda_{i}\right) \sum_{F_{d}} \int_{F_{d}} \frac{\rho^{*} e_{T}\left(U_{d}\right)}{e_{T}\left(N\left(F_{d}\right)\right)\left[\alpha\left(\alpha-c_{1}(L)\right)\right]}
$$

where $\phi_{p_{i}}:=\prod_{j \neq i}\left(p-\lambda_{j}\right), \phi_{\mathbf{P}^{n}}:=\prod_{j=0}^{n} \prod_{m=1}^{d}\left(p-\lambda_{j}-m \alpha\right) \epsilon$ $H_{T}^{*}\left(\mathbf{P}^{n}\right)$. From the localization formula, we deduce that 


$$
\frac{\rho^{*} e_{T}\left(U_{d}\right)}{\alpha\left(\alpha-c_{1}(L)\right) e v^{*} \phi_{p_{i}}=\int_{\mathrm{P}^{n}} e v !\left(\frac{\rho^{*} e_{T}\left(U_{d}\right)}{\alpha\left(\alpha-c_{1}(L)\right)}\right) \phi_{p_{i}}=i_{p_{i}}^{*} e v_{1}\left(\frac{\rho^{*} e_{T}\left(U_{d}\right)}{\alpha\left(\alpha-c_{1}(L)\right)}\right)} \text { Thus(3.1) canbewri }
$$

$\iota_{p_{i}}^{*}\left[\phi_{\mathbf{P}_{n}} \quad e v_{!}\left(\frac{\rho^{*} e_{T}\left(U_{d}\right)}{\alpha\left(\alpha-c_{1}(L)\right)}\right)\right], \quad i=0, . .$, n.Itfollowsthat $I_{d}^{*}\left(Q_{d}\right)=$ $\phi_{\mathbf{P}^{n}} e v_{!}\left(\frac{\rho^{*} e_{T}\left(U_{d}\right)}{\alpha\left(\alpha-c_{1}(L)\right)}\right) \cdot(3.2) T h i s s h o w s t h a t \operatorname{deg}_{\alpha} I_{d}^{*}\left(Q_{d}\right) \leq \operatorname{deg}_{\alpha} \phi_{\mathbf{P}^{n}}-$ $2=(n+1) d-2$, proving (i).

Since $Q_{d}=\varphi_{!} e_{T}\left(V_{d}\right) \in H_{G}^{*}\left(N_{d}\right)$, their nonequivariant limit $\lambda \rightarrow$ 0 exist. In this limit (3.2) gives

$$
\begin{aligned}
A & :=\int_{\mathbf{P}^{n}} e^{-H t / \alpha} \frac{I_{d}^{*}\left(Q_{d}\right)}{\prod_{m=1}^{d}(H-m \alpha)^{n+1}} \\
& =\int_{\overline{\mathcal{M}}_{0,1}\left(d, \mathbf{P}^{n}\right)} e^{-e v^{*} H t / \alpha} \frac{\rho^{*} c_{t o p}\left(U_{d}\right)}{\alpha\left(\alpha-c_{1}(L)\right)} \\
& =\int_{\overline{\mathcal{M}}_{0,0}\left(d, \mathbf{P}^{n}\right)} c_{t o p}\left(U_{d}\right) \rho_{!}\left(\frac{e^{-e v^{*} H t / \alpha}}{\alpha\left(\alpha-c_{1}(L)\right)}\right) .
\end{aligned}
$$

Now $c_{\text {top }}\left(U_{d}\right)$ has degree the same as the dimension of $\overline{\mathcal{M}}_{0,0}\left(d, \mathbf{P}^{n}\right)$. The second factor in the last integrand contributes a scalar factor given by integration over a generic fiber $E$ (which is a $\mathbf{P}^{1}$ ) of $\rho$. So we pick out the degree 1 term in $\frac{e^{-e v^{*} H t / \alpha}}{\alpha\left(\alpha-c_{1}(L)\right)}$, which is just $\frac{-e v^{*} H t}{\alpha^{3}}+\frac{c_{1}(L)}{\alpha^{3}}$. Restricting to the generic fiber $E$, say over $(f, C) \in \overline{\mathcal{M}}_{0,0}\left(d, \mathbf{P}^{n}\right)$, the evaluation map $e v$ is equal to $f$, which is a degree $d$ map $E \cong \mathbf{P}^{1} \rightarrow \mathbf{P}^{n}$. It follows that

$$
\int_{E} e v^{*} H=d
$$

Moreover, since $c_{1}(L)$ restricted to $E$ is just the first Chern class of the tangent bundle to $E$, it follows that

$$
\int_{E} c_{1}(L)=2
$$

So we have

$$
A=\left(-\frac{d t}{\alpha^{3}}+\frac{2}{\alpha^{3}}\right) K_{d}
$$

It is easy to work out the complete list of critical concavex vector bundles $V$ on $\mathbf{P}^{n}$ which are direct sums of line bundles. Such a $V$ is of the form

$$
\begin{aligned}
V & =V^{+} \oplus V^{-} \\
V^{+} & =\oplus_{a=1}^{N^{+}} \mathcal{O}\left(l_{a}\right) \\
V^{-} & =\oplus_{b=1}^{N^{-}} \mathcal{O}\left(-k_{b}\right)
\end{aligned}
$$


where $l_{1}, . ., l_{N^{+}}, k_{1}, . ., k_{N^{-}}$are positive integers. By Riemann-Roch, the bundles $U_{d}$ that $V$ induces on $\overline{\mathcal{M}}_{0,0}\left(d, \mathbf{P}^{n}\right)$ has rank $U_{d}=$ $d\left(\sum l_{a}+\sum k_{b}\right)+N^{+}-N^{-}$, which must be $(n+1) d+n-3$ for all $d$ if $V$ is critical. Thus we must have

$$
\begin{aligned}
\sum l_{a}+\sum k_{b} & =n+1 \\
N^{+}-N^{-} & =n-3 .
\end{aligned}
$$

The complete list of critical bundles that are also direct sums of line bundles is:

$$
\begin{array}{ll}
\mathbf{P}^{1}: & \mathcal{O}(-1) \oplus \mathcal{O}(-1) \\
\mathbf{P}^{2}: & \mathcal{O}(-3) \\
\mathbf{P}^{3}: & \mathcal{O}(2) \oplus \mathcal{O}(-2), \mathcal{O}(3) \oplus \mathcal{O}(-1) \\
\mathbf{P}^{4}: & \mathcal{O}(5), \quad \mathcal{O}(2) \oplus \mathcal{O}(2) \oplus \mathcal{O}(-1) \\
\mathbf{P}^{5}: & \mathcal{O}(2) \oplus \mathcal{O}(4), \quad \mathcal{O}(3) \oplus \mathcal{O}(3) \\
\mathbf{P}^{6}: & \mathcal{O}(2) \oplus \mathcal{O}(2) \oplus \mathcal{O}(3) \\
\mathbf{P}^{7}: & \mathcal{O}(2) \oplus \mathcal{O}(2) \oplus \mathcal{O}(2) \oplus \mathcal{O}(2) .
\end{array}
$$

Note that we have excluded the critical bundles in which the hyperplane bundle $\mathcal{O}(1)$ occurs because in the nonequivariant limit it only reduces a given case of $\mathbf{P}^{n}$ to $\mathbf{P}^{n-1}$. For example, even though the bundle $\mathcal{O}(1) \oplus \mathcal{O}(-1) \oplus \mathcal{O}(-1)$ on $\mathbf{P}^{2}$ is certainly critical, computing the $K_{d}$ for the induced bundles is equivalent to doing the same with $\mathcal{O}(-1) \oplus \mathcal{O}(-1)$ on $\mathbf{P}^{1}$. It is curious to note that the numerical conditions (3.3) is rather similar to the condition for having a projective complete intersection Calabi-Yau threefold. In fact five of the examples on $\mathbf{P}^{4}$ through $\mathbf{P}^{7}$ above involving only positive bundles are exactly the cases in which each critical bundle cuts out a complete intersection Calabi-Yau threefold. We also note that, with the exception of the $\mathbf{P}^{1}$ case, the three examples which involve negative bundles in fact correspond to noncompact Calabi-Yau threefolds. The total space of $\mathcal{O}(-3) \rightarrow \mathbf{P}^{2}$, the total space of $\psi^{*} \mathcal{O}(-2) \rightarrow X$, where $\psi: X \hookrightarrow \mathbf{P}^{3}$ is a quadric, and the total space of $\psi^{*} \mathcal{O}(-1) \rightarrow \mathbf{P}^{4}$ where $\psi: X \hookrightarrow \mathbf{P}^{4}$ is the intersection of two quadrics, all three are noncompact Calabi-Yau. These examples arise in the so-called local mirror symmetry. In the next subsection, we shall compute the Euler classes of the induced bundles for the list above. 


\subsection{The first convex example: The Mirror Conjecture}

Throughout this subsection, we set $l=n+1$, consider the convex bundle $V=\mathcal{O}(l)$ on $\mathbf{P}^{n}$, and fix $\Omega^{V}=l p . P, Q$ shall denote the following two linked Euler data (cf. Theorems 2.8, 2.10.):

$$
\begin{aligned}
& P: P_{d}=\prod_{m=0}^{l d}(l \kappa-m \alpha) \\
& Q: Q_{d}=\varphi ! e_{T}\left(V_{d}\right) .
\end{aligned}
$$

Consider the hypergeometric differential equation

$$
\left(\left(\frac{d}{d t}\right)^{n}-l e^{t}\left(l \frac{d}{d t}+1\right) \cdots\left(l \frac{d}{d t}+n\right)\right) h(t)=0 .
$$

We have seen that a basis $f_{i}, i=0, . ., n-1$, of solutions can be read off from the hypergeometric series (cf. Introduction) in the limit $\lambda \rightarrow 0$ :

$$
\begin{aligned}
H G[\mathcal{I}(P)](t) & =e^{-H t / \alpha} \sum_{d \geq 0} \frac{\prod_{m=0}^{l d}(l H-m \alpha)}{\prod_{m=1}^{d}(H-m \alpha)^{n+1}} e^{d t} \\
& =l H\left(f_{0}-f_{1} \frac{H}{\alpha}+f_{2} \frac{H^{2}}{\alpha^{2}}-\cdots\right) .
\end{aligned}
$$

Recall that

$$
T(t):=\frac{f_{1}}{f_{0}}=t+\frac{g_{1}}{f_{0}}
$$

is the mirror map of Candelas et al, where

$$
f_{0}:=\sum_{d \geq 0} \frac{(l d) !}{(d !)^{n+1}} e^{d t}, \quad g_{1}:=\sum_{d \geq 1} \frac{(l d) !}{(d !)^{l}} \sum_{m=d+1}^{l d} \frac{l}{m} e^{d t} .
$$

Lemma 3.3. In the limit $\lambda \rightarrow 0$, we have $H G[\mathcal{I}(Q)](T(t))=$ $\frac{1}{f_{0}} H G[\mathcal{I}(P)](t)$.

Proof: By expanding in powers of $\alpha^{-1}$ and using the assumption that $l=n+1$, we get

$$
\begin{aligned}
H G[\mathcal{I}(P)](t) & =e^{-p t / \alpha} \sum_{d \geq 0} \frac{\prod_{m=0}^{l d}(l p-m \alpha)}{\prod_{k=0}^{n} \prod_{m=1}^{d}\left(p-\lambda_{k}-m \alpha\right)} e^{d t} \\
& =l p\left[f_{0}-\alpha^{-1}\left(p f_{1}+g_{2} \sum_{k=0}^{n} \lambda_{k}\right)+\cdots\right]
\end{aligned}
$$


where $g_{2}=\sum_{d \geq 1} \frac{(l d) !}{(d !)} \sum_{m=1}^{d} \frac{1}{m} e^{d t}$.

Put $f:=\left(\log f_{0}\right) \alpha+\frac{g_{2}}{f_{0}} \sum_{k=0}^{n} \lambda_{k} \in e^{t} \mathcal{R}\left[\left[e^{t}\right]\right]$. By Lemma 2.15, we have a mirror transformation $\mu$ such that

$$
H G[\mathcal{I}(\tilde{P})](t)=e^{f / \alpha} H G[\mathcal{I}(P)](t)
$$

where $\tilde{P}=\mu(P)$. Substituting (3.6) into (3.7), we get

$$
\begin{aligned}
H G[\mathcal{I}(\tilde{P})](t) & =\left(1+\alpha^{-1} \frac{g_{2}}{f_{0}} \sum_{k=0}^{n} \lambda_{k}+\cdots\right) \\
& \times f_{0}^{-1} l p\left[f_{0}-\alpha^{-1}\left(p f_{1}+g_{2} \sum_{k=0}^{n} \lambda_{k}\right)+\cdots\right] \\
& =l p-\alpha^{-1} l p^{2} \frac{f_{1}}{f_{0}}+\cdots .
\end{aligned}
$$
that

By Lemma 2.15 again, we have a mirror transformation $\nu$ such

$$
H G[\mathcal{I}(\tilde{Q})](t)=H G[\mathcal{I}(Q)]\left(t+\frac{g_{1}}{f_{0}}\right)
$$

where $\tilde{Q}=\nu(Q)$. Since, by Theorem $3.2(\mathrm{i}), I_{d}^{*}\left(Q_{d}\right)=O\left(\alpha^{(n+1) d-2}\right)$, it is straightforward to find that

$$
H G[\mathcal{I}(\tilde{Q})](t)=e^{-p\left(t+\frac{g_{1}}{f_{0}}\right) / \alpha}(l p+\cdots)=l p-\alpha^{-1} l p^{2}\left(t+\frac{g_{1}}{f_{0}}\right)+\cdots
$$

From (3.8) and (3.9), we conclude that for $d>0$,

$$
\frac{I_{d}^{*}\left(\tilde{P}_{d}-\tilde{Q}_{d}\right)}{\prod_{k=0}^{n} \prod_{m=1}^{d}\left(p-\lambda_{k}-m \alpha\right)} \equiv 0
$$

modulo order $\alpha^{-2}$, and hence

$$
\operatorname{deg}_{\alpha} \iota_{p_{i, 0}}^{*}\left(\tilde{P}_{d}-\tilde{Q}_{d}\right) \leq(n+1) d-2 .
$$

But $\tilde{P}=\mu(P)$ is linked to $P$, and $\tilde{Q}=\nu(Q)$ is linked to $Q$. Since $P$ and $Q$ are linked, it follows that $\tilde{P}$ and $\tilde{Q}$ are also linked. By Theorem 2.11 , we have $\tilde{P}=\tilde{Q}$. In particular, we have

$$
H G[\mathcal{I}(Q)](T(t))=H G[\mathcal{I}(\tilde{Q})](t)=H G[\mathcal{I}(\tilde{P})](t)=e^{f / \alpha} H G[\mathcal{I}(P)](t)
$$


Since both $P_{d}, Q_{d}$ lie in $H_{G}^{*}\left(N_{d}\right)$, their nonequivariant limit exist. Taking $\lambda \rightarrow 0$ yields our assertion.

Throughout the rest of this subsection, we set $l=n+1=5$ and consider the critical bundle $\mathcal{O}(5) \rightarrow \mathbf{P}^{4}$. We assume that we have taken the nonequivariant limit $\lambda \rightarrow 0$. Recall that

$$
F(T):=\frac{5 T^{3}}{6}+\sum_{d>0} K_{d} e^{d T}, \quad \mathcal{F}(T):=\frac{5}{2}\left(\frac{f_{1}}{f_{0}} \frac{f_{2}}{f_{0}}-\frac{f_{3}}{f_{0}}\right) .
$$

Theorem 3.4. (The Mirror Conjecture) $F=\mathcal{F}$.

Proof: Since

$$
H G[\mathcal{I}(P)](t)=5 H\left(f_{0}-f_{1} \frac{H}{\alpha}+f_{2} \frac{H^{2}}{\alpha^{2}}-f_{3} \frac{H^{3}}{\alpha^{3}}\right),
$$

we will prove that (cf. [8])

$$
H G[\mathcal{I}(Q)](T)=5 H\left(1-T \frac{H}{\alpha}+\frac{F^{\prime}}{5} \frac{H^{2}}{\alpha^{2}}-\frac{T F^{\prime}-2 F}{5} \frac{H^{3}}{\alpha^{3}}\right) .
$$

Eqns. (3.10), (3.11) and the preceding lemma imply $F=\mathcal{F}$. Denote the right hand side of (3.11) by $R$. Then

$$
e^{H T / \alpha} R=5 H\left(1+\frac{\Phi^{\prime}}{5} \frac{H^{2}}{\alpha^{2}}+\frac{2 \Phi}{5} \frac{H^{3}}{\alpha^{3}}\right)=5 H+O\left(e^{T}\right)
$$

where $\Phi:=F-\frac{5 T^{3}}{6}=O\left(e^{T}\right)$. Similarly $e^{H T / \alpha} H G[\mathcal{I}(Q)](T)=5 H+$ $O\left(e^{T}\right)$, which also has no polynomial dependence on $T$. So (3.11) is equivalent to

$$
\int_{\mathbf{P}^{4}} e^{-H T / \alpha}\left(e^{H T / \alpha} H G[\mathcal{I}(Q)]\right)=\int_{\mathbf{P}^{4}} e^{-H T / \alpha}\left(e^{H T / \alpha} R\right) .
$$

By Theorem 3.2(ii), this left hand side is

$$
\int_{\mathbf{P}^{4}} H G[\mathcal{I}(Q)]=\alpha^{-3}\left(2 \Phi-T \Phi^{\prime}\right)+\int_{\mathbf{P}^{4}} e^{-H T / \alpha} 5 H=\alpha^{-3}\left(2 F-T F^{\prime}\right),
$$

which coincides with $\int_{\mathbf{P}^{4}} R$. 
It is straightforward to generalize Theorem 3.4 to all other critical convex bundles $V$ in the list (3.4). In each case, $\Omega^{V}$ becomes $\prod_{a} l_{a} p$ and the Euler data $P$ to be linked with $Q: Q_{d}:=\varphi_{!} e_{T}\left(V_{d}\right)$ is given by $P_{d}=\prod_{a} \prod_{m=0}^{l_{a} d}\left(l_{a} \kappa-m \alpha\right)$. In the nonequivariant limit, the hypergeometric series $H G[\mathcal{I}(P)](t)$ will produce some hypergeometric functions $f_{0}, . ., f_{3}$ defining the function $\mathcal{F}$. The generating function $F$ for the $K_{d}$ is modified by simply replacing the term $\frac{5 T^{3}}{6}$ by $\frac{T^{3}}{6} \int_{X} \psi^{*} H^{3}$, where $\psi: X \rightarrow \mathbf{P}^{n}$ is the Calabi-Yau cut out by $V$. With these minor modifications in each case, Theorem 3.4 holds. We leave the details as an exercise for the reader.

\subsection{First concave example: multiple-cover formula}

Let $V$ be the bundle $\mathcal{O}(-1) \oplus \mathcal{O}(-1)$ on $\mathbf{P}^{1}$. For $d>1, V$ induces a rank $2 d-2$ bundle $U_{d} \rightarrow \mathcal{M}_{0,0}\left(d, \mathbf{P}^{1}\right)$ whose fiber at $(f, C)$ is the space $H^{1}\left(C, f^{*} V\right)$, thus $V$ is a critical concave bundle on $\mathbf{P}^{1}$. We set $\Omega^{V}=1 / e_{T}(V)=p^{-2}$. We shall compute the equivariant classes $Q_{d}:=\varphi_{!} e_{T}\left(V_{d}\right)$, and the numbers $K_{d}$ for this critical bundle. Note that by definition $Q_{1}=1$ and $K_{1}=1$. As a consequence of Theorem 2.10 ,

$$
\iota_{p_{i, 0}}^{*}\left(Q_{d}\right)=\prod_{m=1}^{d-1}\left(\lambda_{i}-m\left(\lambda_{i}-\lambda_{j}\right) / d\right)^{2}
$$

at $\alpha=\left(\lambda_{i}-\lambda_{j}\right) / d, j \neq i$. Thus $Q$ is linked to

$$
P: \quad P_{d}:=\prod_{m=1}^{d-1}(\kappa-m \alpha)^{2}
$$

which is a $p^{-2}$-Euler data. Obviously $\operatorname{deg}_{\alpha} I_{d}^{*}\left(P_{d}\right)=2 d-2$. It follows from Theorem 3.2 (i) that $\operatorname{deg}_{\alpha} I_{d}^{*}\left(P_{d}-Q_{d}\right) \leq 2 d-2$, implying $Q=P$ by Theorem 2.11 .

Corollary 3.5. $K_{d}=d^{-3}$.

Proof: By Theorem 3.2(ii) in the limit $\lambda \rightarrow 0$, we have

$$
\int_{\mathbf{P}^{1}} e^{-H t / \alpha} \frac{I_{d}^{*}\left(Q_{d}\right)}{\prod_{m=1}^{d}(H-m \alpha)^{2}}=\alpha^{-3}(2-d t) K_{d} .
$$

Since $Q=P$, we have $I_{d}^{*}\left(Q_{d}\right)=I_{d}^{*}\left(P_{d}\right)=\prod_{m=1}^{d-1}(H-m \alpha)^{2}$, giving

$$
\int_{\mathbf{P}^{1}} e^{-H t / \alpha} \frac{I_{d}^{*}\left(Q_{d}\right)}{\prod_{m=1}^{d}(H-m \alpha)^{2}}=\alpha^{-3} d^{-3}(2-d t) .
$$




\subsection{Second concave example: $K_{\mathrm{P}^{2}}$}

Let $V$ be the canonical bundle $\mathcal{O}(-3) \rightarrow \mathbf{P}^{2}$. For $d>0$, this bundle induces a rank $3 d-1$ bundle $U_{d} \rightarrow \overline{\mathcal{M}}_{0,0}\left(d, \mathbf{P}^{2}\right)$. Thus $V$ is a critical concave bundle. We set $\Omega^{V}=1 / e_{T}(V)=(-3 p)^{-1}$. We shall compute the equivariant classes $Q_{d}:=\varphi_{!} e_{T}\left(V_{d}\right)$, and the numbers $K_{d}$ for this critical bundle. As a consequence of Theorem 2.10, we have

$$
\iota_{p_{i, 0}}^{*}\left(Q_{d}\right)=\prod_{m=1}^{3 d-1}\left(-3 \lambda_{i}+m\left(\lambda_{i}-\lambda_{j}\right) / d\right)
$$

at $\alpha=\left(\lambda_{i}-\lambda_{j}\right) / d, j \neq i$. Thus $Q$ is linked to

$$
P: \quad P_{d}:=\prod_{m=1}^{3 d-1}(-3 \kappa+m \alpha)
$$

which is a $(-3 p)^{-1}$-Euler data.

Corollary 3.6. $H G[\mathcal{I}(Q)](t+g)=H G[\mathcal{I}(P)](t)$ where $g:=$ $\sum_{d>0} \frac{(-1)^{d}}{d} \frac{(3 d) !}{d !^{3}} e^{d t}$.

Proof: By expanding in powers of $\alpha^{-1}$, we get

$$
\begin{aligned}
H G[\mathcal{I}(P)](t) & =e^{-p t / \alpha} \\
& \times\left((-3 p)^{-1}+\sum_{d>0} \frac{\prod_{m=1}^{3 d-1}(-3 p+m \alpha)}{\prod_{k=0}^{2} \prod_{m=1}^{d}\left(p-\lambda_{k}-m \alpha\right)} e^{d t}\right) \\
& =(-3 p)^{-1}+\alpha^{-1} \frac{t+g}{3}+\cdots
\end{aligned}
$$

As before, it is now straightforward to show that

$$
H G[\mathcal{I}(Q)](t+g) \equiv H G[\mathcal{I}(P)](t)
$$

modulo order $\alpha^{-2}$. Once again by Theorem 2.11, the two sides are equal identically.

Using Theorem 3.2(ii) and the preceding corollary, we obtain the 
$K_{d}$, for $d=1, . ., 10$ :

\begin{tabular}{|r|r|}
\hline$d$ & $K_{d}$ \\
\hline 1 & 3 \\
2 & $-\frac{45}{8}$ \\
3 & $\frac{244}{9}$ \\
4 & $-\frac{12333}{64}$ \\
5 & $\frac{211878}{125}$ \\
6 & $-\frac{102365}{6}$ \\
7 & $\frac{64639725}{343}$ \\
8 & $-\frac{1140830253}{512}$ \\
9 & $\frac{6742982701}{243}$ \\
10 & $-\frac{36001193817}{100}$ \\
\hline
\end{tabular}

3.4. A concavex bundle on $\mathbf{P}^{3}$.

Let $V=\mathcal{O}(2) \oplus \mathcal{O}(-2)$ on $\mathbf{P}^{3}$. This is a direct sum of a convex and a concave bundle. The induced bundle $U_{d} \rightarrow \bar{M}_{0,0}\left(d, \mathbf{P}^{3}\right)$, with fiber at $(f, C)$ being $H^{0}\left(C, f^{*} \mathcal{O}(2)\right) \oplus H^{1}\left(C, f^{*} \mathcal{O}(-2)\right)$, has rank $4 d$. We set $\Omega^{V}=e_{T}(\mathcal{O}(2)) / e_{T}(\mathcal{O}(-2))=-1$. We shall compute the equivariant classes $Q_{d}:=\varphi_{!} e_{T}\left(V_{d}\right)$, and the numbers $K_{d}$ for this critical bundle. As a consequence of Theorem 2.10, we have

$$
\iota_{p_{i, 0}}^{*}\left(Q_{d}\right)=\prod_{m=0}^{2 d}\left(2 \lambda_{i}-m\left(\lambda_{i}-\lambda_{j}\right) / d\right) \times \prod_{m=1}^{2 d-1}\left(-2 \lambda_{i}+m\left(\lambda_{i}-\lambda_{j}\right) / d\right)
$$

at $\alpha=\left(\lambda_{i}-\lambda_{j}\right) / d, j \neq i$. Thus $Q$ is linked to

$$
P: \quad P_{d}:=\prod_{m=0}^{2 d}(2 \kappa-m \alpha) \times \prod_{m=1}^{2 d-1}(-2 \kappa+m \alpha)
$$

which is a -1-Euler data.

Corollary 3.7. $H G[\mathcal{I}(Q)](t+g)=H G[\mathcal{I}(P)](t)$ where $g:=$ 
$\sum_{d>0} \frac{1}{d} \frac{(2 d) !^{2}}{d !^{4}} e^{d t}$

Proof: By expanding in powers of $\alpha^{-1}$, we get

$$
\begin{aligned}
H G[\mathcal{I}(P)](t) & =e^{-p t / \alpha} \\
& \times\left(-1+\sum_{d>0} \frac{\prod_{m=0}^{2 d}(2 p-m \alpha) \times \prod_{m=1}^{2 d-1}(-2 p+m \alpha)}{\prod_{k=0}^{3} \prod_{m=1}^{d}\left(p-\lambda_{k}-m \alpha\right)} e^{d t}\right) \\
& =-1+\alpha^{-1} p(t+g)+\cdots,
\end{aligned}
$$

which, as in the previous examples, agrees with $H G[\mathcal{I}(Q)](t+g)$ up to order $\alpha^{-2}$. Hence Theorem 2.11 yields our assertion.

Using Theorem 3.2(ii) and the preceding corollary, we obtain the $K_{d}$, for $d=1, . ., 10$ :

\begin{tabular}{|r|r|}
\hline$d$ & $K_{d}$ \\
\hline 1 & -4 \\
2 & $-\frac{9}{2}$ \\
3 & $-\frac{328}{27}$ \\
4 & $-\frac{777}{16}$ \\
5 & $-\frac{30004}{125}$ \\
6 & $-\frac{4073}{3}$ \\
7 & $-\frac{2890808}{343}$ \\
8 & $-\frac{7168777}{128}$ \\
9 & $-\frac{285797488}{729}$ \\
10 & $-\frac{714787509}{250}$ \\
\hline
\end{tabular}

3.5. A concavex bundle on $\mathbf{P}^{4}$.

Consider now the critical bundle $V=\mathcal{O}(2) \oplus \mathcal{O}(2) \oplus \mathcal{O}(-1)$ on $\mathbf{P}^{4}$. The induced bundle $U_{d} \rightarrow \overline{\mathcal{M}}_{0,0}\left(d, \mathbf{P}^{3}\right)$, has rank $5 d+1$. We set $\Omega^{V}=e_{T}(\mathcal{O}(2))^{2} / e_{T}(\mathcal{O}(-1))=-4 p$. We shall compute the equivariant classes $Q_{d}:=\varphi_{!} e_{T}\left(V_{d}\right)$, and the numbers $K_{d}$ for this critical bundle. As a consequence of Theorem 2.10, we have

$$
\iota_{p_{i, 0}}^{*}\left(Q_{d}\right)=\prod_{m=0}^{2 d}\left(2 \lambda_{i}-m\left(\lambda_{i}-\lambda_{j}\right) / d\right)^{2} \times \prod_{m=1}^{d-1}\left(-\lambda_{i}+m\left(\lambda_{i}-\lambda_{j}\right) / d\right) .
$$


at $\alpha=\left(\lambda_{i}-\lambda_{j}\right) / d, j \neq i$. Thus $Q$ is linked to

$$
P: \quad P_{d}:=\prod_{m=0}^{2 d}(2 \kappa-m \alpha)^{2} \times \prod_{m=1}^{d-1}(-\kappa+m \alpha) .
$$

which is a $-4 p$-Euler data.

Corollary 3.8. $H G[\mathcal{I}(Q)](t+g)=H G[\mathcal{I}(P)](t)$ where $g:=$ $\sum_{d>0} \frac{(-1)^{d}}{d} \frac{(2 d) !^{2}}{d !^{4}} e^{d t}$

Proof: By expanding in powers of $\alpha^{-1}$, we get

$$
\begin{aligned}
H G[\mathcal{I}(P)](t) & =e^{-p t / \alpha}\left(-4 p+\sum_{d>0} \frac{\prod_{m=0}^{2 d}(2 p-m \alpha)^{2} \times \prod_{m=1}^{d-1}(-p+m \alpha)}{\prod_{k=0}^{4} \prod_{m=1}^{d}\left(p-\lambda_{k}-m \alpha\right)} e^{d t}\right) \\
& =-4 p+\alpha^{-1} 4 p^{2}(t+g)+\cdots,
\end{aligned}
$$

which, as in the previous examples, agrees with $H G[\mathcal{I}(Q)](t+g)$ up to order $\alpha^{-2}$. Hence Theorem 2.11 yields our assertion.

We can work out the $K_{d}$ here as we did before. The $K_{d}$ here can be obtained by taking $K_{d}$ from the preceding example on $\mathrm{P}^{3}$, and multiply it by $4(-1)^{d}$. This is so because in the nonequivariant limit, the hypergeometric series $H G[\mathcal{I}(P)](t)$ (cf. (3.13) and (3.14)) in this example on $\mathrm{P}^{4}$ and the preceding example on $\mathrm{P}^{3}$ are related by first a multiplication of $4 p$ followed by a change of variable $e^{d t} \mapsto(-1)^{d} e^{d t}$.

\subsection{General concavex bundles}

In fact the examples above are representative of the most general concavex bundle. Let $V=V^{+} \oplus V^{-}$be a concavex bundle on $\mathrm{P}^{n}$, and let $Q, P$ be as defined in Theorem 2.10, and assume that $V$ has splitting type $\left(l_{1}, . ., l_{N^{+}} ; k_{1}, . ., k_{N^{-}}\right)$. Note that $\sum l_{a}+\sum k_{b}$ is the value of the class $c_{1}\left(V^{+}\right)-c_{1}\left(V^{-}\right)$on a $T$-invariant $\mathbf{P}^{1}$ in $\mathbf{P}^{n}$.

Theorem 3.9. If $d\left(\sum l_{a}+\sum k_{b}\right)-N^{-} \leq(n+1) d-2$ for all $d>0$, then $Q=P$. If $d\left(\sum l_{a}+\sum k_{b}\right)-N^{-} \leq(n+1) d$ for all $d>0$, then there exists a mirror transformation $\mu$, depending only on the $l_{a}, k_{b}$, such that $Q=\mu(P)$.

Proof: By definition of $P$ in Theorem 2.10,

$$
\operatorname{deg}_{\alpha} I_{d}^{*}\left(P_{d}\right)=d\left(\sum l_{a}+\sum k_{b}\right)-N^{-}
$$


Consider the first case, where this is bounded above by $(n+1) d-2$ for all $d$. Then by Theorem 3.2,

$$
\operatorname{deg}_{\alpha}\left(I_{d}^{*}\left(P_{d}\right)-I_{d}^{*}\left(Q_{d}\right)\right) \leq(n+1) d-2,
$$

implying $Q=P$ by Theorem 2.11 .

Consider now the second case. Obviously our assumption implies that $\sum l_{a}+\sum k_{b} \leq(n+1)$. It is trivial to show that the only possibilities not covered by the first case are: (1) $N^{-}=0$ and $\sum l_{a}=n+1$; (2) $N^{-}=1$ and $\sum l_{a}+k_{1}=n+1$. (3) $N^{-}=0$ and $\sum l_{a}=n$; In each of these cases, a mirror transformation can be constructed by immitating the previous examples in a straightforward way. Case (1) immitates the example $\mathcal{O}(5) \rightarrow \mathbf{P}^{4}$, while cases (2), (3) immitate the example $\mathcal{O}(-3) \rightarrow \mathbf{P}^{2}$. It is obvious that in each case, the mirror transformation depends only on the data $l_{a}, k_{b}$.

Corollary 3.10. Under the same hypotheses as in the preceding theorem, the Euler data $Q: Q_{d}=\varphi_{!} e_{T}\left(V_{d}\right)$ depends only on the splitting type, ie. the numbers $l_{a}, k_{b}$, of the concavex bundle $V$ on $\mathbf{P}^{n}$.

Note that not every concavex bundle on $\mathbf{P}^{n}$ is a direct sum of line bundles. For example the tangent bundle is convex, but is not a direct sum of line bundles.

\subsection{Equivariant total Chern class}

For simplicity, we restrict to convex bundles. Let $V$ be a rank $r$ convex bundle on $\mathbf{P}^{n}$, and let

$$
c_{T}(V)=x^{r}+x^{r-1} c_{1}(V)+\cdots+c_{r}(V)
$$

be the $T$-equivariant Chern polynomial of $V$. Similarly we denote by $c_{T}\left(U_{d}\right)$ the equivariant Chern polynomial for $U_{d}$. As explained in Example 9, we can extend the notion of Euler data $Q$ allowing $Q_{d}$ to depend on $x$ polynomially, simply by replacing the ground field $\mathbf{Q}$ by $\mathbf{Q}(x)$. Then a similar argument as in Theorem 2.8 shows that the sequence $Q_{d}:=\varphi_{!}\left(\pi^{*} c_{T}\left(U_{d}\right)\right)$ is also an Euler data in the generalized sense. Moreover, the analogue of Theorem 2.10 holds, ie. at $\alpha=\left(\lambda_{j}-\lambda_{i}\right) / d$, we have the form

$$
\iota_{p_{j, 0}}^{*}\left(Q_{d}\right)=\prod_{a} \prod_{m=0}^{l_{a} d}\left(x+l_{a} \lambda_{j}-m\left(\lambda_{j}-\lambda_{i}\right) / d\right) .
$$


Hence $Q$ is linked to the Euler data

$$
P: \quad P_{d}=\prod_{a} \prod_{m=0}^{l_{a} d}\left(x+l_{a} \kappa-m \alpha\right) .
$$

Again, under a suitable bound on $c_{1}(V)$, one can easily relate $P, Q$ by a generalized (depending on $x$ ) mirror transformation.

For example, by taking the bundle $\mathcal{O}(4)$ on $\mathbf{P}^{3}$, and applying the above result, we can compute the nonequivariant limits of all $\int_{\overline{\mathcal{M}}_{0,0}\left(d, \mathbf{P}^{3}\right)} c_{4 d}\left(U_{d}\right)$. They are expected to count rational curves in a $\mathbf{P}^{1}$-family of K3 hypersurfaces in $\mathbf{P}^{3}$. Similarly we can take $\mathcal{O}(3)$ on $\mathbf{P}^{2}$ and compute $\int_{\overline{\mathcal{M}}_{0,0}\left(d, \mathbf{P}^{2}\right)} c_{3 d-1}\left(U_{d}\right)$ which should count the number of rarional curves in a $\mathbf{P}^{2}$-family of elliptic curves in $\mathbf{P}^{2}$. Details will be reported in full in our forth-coming paper [37].

\subsection{Concluding remarks}

The most important result we establised in this paper is the Mirror Principle. For simplicity we have restricted our examples in this paper, to studying only Euler classes and total Chern classes. As mentioned in the Introduction, the Mirror Principle works well for any multiplicative equivariant characteristic classes. We shall study in details more examples of the total Chern class in our forthcoming paper [37]. Generalization to manifolds with torus action will also be dealt with in details there.

Finally, we make a tantalizing observation which might be of both physical and mathematical significance. As we have seen, the set of linked Euler data has an infinite dimensional transformation group - the mirror group. For suitable concavex bundle $V \rightarrow \mathbf{P}^{n}$, two special linked Euler data (cf. Theorem 2.10) $Q: Q_{d}=\varphi_{!} e_{T}\left(V_{d}\right)$ arising from the nonlinear sigma model (the stable map moduli), and $P$ the corresponding Euler data of hypergeometric type, are related by a mirror transformation. Since the mirror group is so big, there are many other Euler data which are linked to $P$ and can be obtained simply by acting on $P$ by the mirror group. From the physical point of view, $P$ arises from type IIB string theory while $Q$ arises from type IIA string theory, and mirror symmetry is a duality between the two. This relationship manifests itself on the linear sigma model as a duality transformation. This suggests that some other Euler data linked to $P$ may arise from some other string theories which are dual to type IIA and IIB, via more general mirror transformations. From the point of view of moduli theory, $P$ is associated to the linear sigma model compatification $N_{d}$ of the moduli space $M_{d}^{0}$ we discussed in 
Example 10. Whereas $Q$ is associated to the nonlinear sigma model $M_{d}$, which is the stable map compactification of $M_{d}^{0}$. This suggests that some other Euler data linked to $P$ may correspond to other compactifications of $M_{d}^{0}$. If true, we will have an association between string theories, linked Euler data, and compactifications of moduli space of maps, all in the same picture, whereby there is a duality in each kind which one sees in the linear sigma model. It would be interesting to understand this duality more precisely. 


\section{References}

[1] P. Aspinwall and D. Morrison, Topological field theory and rational curves, Commun. Math. Phys. 151 (1993), 245-262.

[2] J. Bryan and N. Leung, The enumerative geometry of K3 surfaces and modular forms, alg-geom/9711031.

[3] M. Atiyah and R. Bott, The moment map and equivariant cohomology, Topology 23 (1984) 1-28.

[4] V. Batyrev and L. Borisov, On Calabi-Yau complete intersections in toric varieties, alg-geom/9412017.

[5] V. Batyrev and D. van Straten, Generalized hypergeometric functions and rational curves on Calabi-Yau complete intersections in toric varieties, Comm. Math. Phys. 168 (1995) 495-533.

[6] M. Bershadsky, S. Cecotti, H. Ooguri, C. Vafa, Kodaira-Spencer theory of gravity and exact results for quantum string amplitude, Commun. Math. Phys. 165 (1994) 311-428.

[7] R. Bott, A residue formula for holomorphic vector fields, J. Diff. Geom. 1 (1967) 311-330.

[8] P. Candelas, X. de la Ossa, P. Green, and L. Parkes, A pair of Calabi-Yau manifolds as an exactly soluble superconformal theory, Nucl. Phys. B359 (1991) 21-74.

[9] P. Candelas, X. de la Ossa, A. Font, S. Katz and D. Morrison, Mirror symmetry for two parameter models I, hep-th/9308083.

[10] L. Caporaso and J. Harris, Parameter spaces for curves on surfaces and enumeration of rational curves, alg-geom/9608024.

[11] B. Crauder and R. Miranda, Quantum cohomology of rational surfaces, Progress in Mathematics 129, Birkhauser (1995) 33.

[12] P. DiFrancesco and C. Itzykson, Quantum intersection rings, Progress in Mathematics 129, Birkhauser (1995) 81.

[13] R. Dijkgraaf, Mirror symmetry and elliptic curves, Progress in Mathematics 129, Birkhauser (1995) 149.

[14] Essays on Mirror Manifolds, ed. S.T. Yau, International Press.

[15] G. Ellingsrud and S.A. Stromme, Bott's formula and enumerative geometry, JAMS 9 (1996), no. 1, 175-193. 
[16] W. Fulton and R. Pandharipande, Notes on stable maps and quantum cohomology, alg-geom/9608011.

[17] E. Getzler, The elliptic Gromov-Witten invariants on $C P^{3}$, alggeom/9612009.

[18] A. Givental, Equivariant Gromov-Witten invariants, alg-geom/9603021.

[19] A. Givental, A mirror theorem for toric complete intersections, alg-geom/9701016.

[20] T. Graber and R. Pandharipande, Localization of Virtual classes, alg-geom/9708001.

[21] B. Greene and R. Plesser, Duality in Calabi-Yau moduli space, Nucl. Phys. B338 (1990) 15-37.

[22] R. Hartshorne, Algebraic Geometry. Graduate Texts in Mathematics 52. Berlin, Heidelberg, New York Springer 1977

[23] F. Hirzebruch, Topological methods in algebraic geometry, SpringerVerlag, Berlin 1995, 3rd Ed.

[24] $\mathrm{Y} . \mathrm{Hu}$, Moduli spaces of stable polygons and symplectic structures on on $\overline{\mathcal{M}}_{0, n}$, Comp. Math. (to appear), alg-geom/9701011.

[25] M. Jinzenji and M. Nagura, Mirror symmetry and exact calculation of $N-2$ point correlation function on Calabi-Yau manifold embedded in $C P^{N-1}$, hep-th/9409029.

[26] M. Kapranov, Chow quotients of Grassmannian, I.M. Gel'fand Seminar, AMS (1993).

[27] B. Kim, On equivariant quantum cohomology, IMRN 17 (1996) 841.

[28] F. Knudsen, and D. Mumford, The projectivity of the moduli space of stable curves I, preliminaries on 'det' and 'div'. Math. Scand. 39 (1976) 19-55.

[29] D. Morrison and R. Plesser, Summing the instantons: quaritum cohomology and mirror symmetry in toric varieties, alggeom/9412236.

[30] S. Hosono, A. Klemm, S. Theisen and S.T. Yau, Mirror symmetry, mirror map and applications to complete intersection CalabiYau spaces, hep-th/9406055. 
[31] S. Hosono, B.H. Lian and S.T. Yau, GKZ-generalized hypergeometric systems and mirror symmetry of Calabi-Yau hypersurfaces, alg-geom/9511001, Commun. Math. Phys. 182 (1996) 535578.

[32] W.Y. Hsiang, On characteristic classes and the topological Schur lemma from the topological transformation groups viewpoint, Proc. Symp. Pure Math. XXII. (1971) 105-112.

[33] S. Katz, On the finiteness of rational curves on quintic threefolds, Comp. Math. 60 (1986) 151-162.

[34] M. Kontsevich, Enumeration of rational curves via torus actions. In: The Moduli Space of Curves, ed. by RDijkgraaf, CF́aber, Gìan der Geer, Progress in Mathvioli29, Birkhäuser, 1995, 335368.

[35] J. Li, Algebraic geometric interpretation of Donaldson's polynomial invariants, Journ. Diff. Geom. 37 (1993) 417-466.

[36] A. Libgober and J. Teitelboim, Duke Math. Journ., Int. Res. Notices 1 (1993) 29.

[37] B. Lian, K. Liu, and S.T. Yau, Mirror Principle II, in preparation.

[38] Yu.I. Manin, Generating functions in algebraic geometry and sums over trees. In: The Moduli Space of Curves, ed. by RDijkgraaf, CF́aber, Givan der Geer, Progress in Mathvioli29, Birkhäuser, 1995, 401-418.

[39] Yu.I. Manin, Frobenius Manifolds, quantum cohomology, and moduli spaces (Chapter I,II,III)., Max-Planck Inst. preprint MPI 96-113.

[40] D. Morrison, Picard-Fuchs Equations and Mirror Maps for Hypersurfaces, in [14].

[41] C. Okonek, M. Schneider and H. Spindler Vector bundles on complex projective spaces, Progress in Math., Birkhauser.

[42] Y.B. Ruan and G. Tian, A mathematical theory of quantum cohomology, Journ. Diff. Geom. Vol. 42, No. 2 (1995) 259-367.

[43] G. Tian, private communication, November 1995.

[44] C. Voisin, A mathematical proof of a formula of AspinwallMorrison, Comp. Math. 104 (1996) 135-151. 
[45] E. Witten, Phases of $N=2$ theories in two dimension, hepth/9301042. 